\title{
Universal Recovery Maps and Approximate Sufficiency of Quantum Relative Entropy
}

\author{
Marius Junge, Renato Renner, David Sutter, Mark M. Wilde \\ and Andreas Winter
}

\begin{abstract}
The data processing inequality states that the quantum relative entropy between two states $\rho$ and $\sigma$ can never increase by applying the same quantum channel $\mathcal{N}$ to both states. This inequality can be strengthened with a remainder term in the form of a distance between $\rho$ and the closest recovered state $(\mathcal{R} \circ \mathcal{N})(\rho)$, where $\mathcal{R}$ is a recovery map with the property that $\sigma=(\mathcal{R} \circ \mathcal{N})(\sigma)$. We show the existence of an explicit recovery map that is universal in the sense that it depends only on $\sigma$ and the quantum channel $\mathcal{N}$ to be reversed. This result gives an alternate, information-theoretic characterization of the conditions for approximate quantum error correction.
\end{abstract}

\section{Introduction}

For two Hilbert spaces $A$ and $B$, let $\mathrm{S}(A)$ denote the set of density operators acting on $A$ and let $\operatorname{TPCP}(A, B)$ be the set of trace-preserving completely positive maps from $A$ to $B .{ }^{1}$ Let $\mathrm{Q}(A)$ denote some subset of $\mathrm{S}(A)$. A quantum channel $\mathcal{N} \in \operatorname{TPCP}(A, B)$ is called sufficient (or reversible) with respect to $\mathrm{Q}(A)$, if there exists a recovery map $\mathcal{R} \in \operatorname{TPCP}(B, A)$ such that

$$
(\mathcal{R} \circ \mathcal{N})(\rho)=\rho \text { for all } \rho \in \mathrm{Q}(A) .
$$

Sufficiency of quantum channels has been studied extensively (see [16,26, 29,30] and references therein).

\footnotetext{
${ }^{1}$ A linear mapping $\mathcal{N}_{A \rightarrow B}$ from $A$ to $B$ is said to be completely positive if $\left(\mathcal{N}_{A \rightarrow B} \otimes\right.$ $\left.\mathcal{I}_{R}\right)\left(\rho_{A R}\right) \geq 0$ for all $\rho_{A R} \geq 0$, where $R$ denotes an arbitrary reference system and $\mathcal{I}_{R}$ denotes the identity map on $R$. The mapping is additionally trace preserving if any positive semi-definite trace-class input operator is mapped to an output operator that has the same trace.
} 

as $[22]$

The quantum relative entropy between two states $\rho$ and $\sigma$ is defined

$$
\begin{aligned}
D(\rho \| \sigma) & :=\sum_{i}\left\langle\phi_{i}|\rho(\log \rho-\log \sigma)| \phi_{i}\right\rangle \\
& =\sum_{i, j}\left|\left\langle\phi_{i} \mid \psi_{j}\right\rangle\right|^{2}[p(i) \log p(i)-p(i) \log q(j)]
\end{aligned}
$$

where $\rho=\sum_{i} p(i)\left|\phi_{i}\right\rangle\left\langle\phi_{i}\right|$ and $\sigma=\sum_{j} q(j)\left|\psi_{j}\right\rangle\left\langle\psi_{j}\right|$ are spectral decompositions of $\rho$ and $\sigma$ with $\left\{\left|\phi_{i}\right\rangle\right\}_{i}$ and $\left\{\left|\psi_{j}\right\rangle\right\}_{j}$ orthonormal bases. Note that if the support of $\rho$ is not contained in the support of $\sigma$, then $D(\rho \| \sigma)=+\infty$. The data processing inequality (also known as monotonicity of the relative entropy) states that $D(\rho \| \sigma)$ is non-increasing under trace-preserving completely positive maps $[24,41]$, i.e.,

$$
D(\rho \| \sigma) \geq D(\mathcal{N}(\rho) \| \mathcal{N}(\sigma))
$$

where $\mathcal{N}$ is a quantum channel. The data processing inequality is related to the sufficiency of $\mathcal{N}$. As shown in $[16,17,29,30]$, a quantum channel $\mathcal{N} \in \operatorname{TPCP}(A, B)$ is sufficient with respect to $\mathrm{Q}(A)$ if and only if $D(\rho \| \sigma)=$ $D(\mathcal{N}(\rho) \| \mathcal{N}(\sigma))$ for all $\rho \in \mathrm{Q}(A)$ and $\sigma \in \mathrm{Q}(A)$. It is known that this is the case if and only if there exists a recovery map $\mathcal{R} \in \operatorname{TPCP}(B, A)$ that simultaneously reverses the action of the physical evolution $\mathcal{N}$ on both states [29-31], i.e., $(\mathcal{R} \circ \mathcal{N})(\rho)=\rho$ and $(\mathcal{R} \circ \mathcal{N})(\sigma)=\sigma$.

Let $\mathrm{L}(B)$ denote the set of bounded operators on $B, \mathrm{TC}(A)$ the set of trace-class operators acting on $A$, and $\mathcal{N}^{\dagger}$ the adjoint map of $\mathcal{N}^{2}$ We consider $\left\langle a_{1}, a_{2}\right\rangle_{\omega}:=\operatorname{tr}\left(a_{1}^{\dagger} \omega^{\frac{1}{2}} a_{2} \omega^{\frac{1}{2}}\right)$, which is an inner product on the space of operators $\left\{a \in \mathrm{L}(A): \Pi_{\omega} a=a \Pi_{\omega}=a\right\}$, where $\Pi_{\omega}$ is the projection onto the support of $\omega$. If the Hilbert spaces $A$ and $B$ are assumed to be separable and $\sigma \in \mathrm{TC}(A)$ is positive semi-definite, then the recovery map can be taken as the Petz recovery map $\mathcal{P}_{\sigma, \mathcal{N}}$ (also known as the transpose map), defined as the adjoint of the solution to

$$
\left\langle a, \mathcal{N}^{\dagger}(b)\right\rangle_{\sigma}=\left\langle\mathcal{P}_{\sigma, \mathcal{N}}^{\dagger}(a), b\right\rangle_{\mathcal{N}(\sigma)} \quad \text { for all } \quad a \in \mathrm{L}(A), b \in \mathrm{L}(B) .
$$

The Petz recovery map $\mathcal{P}_{\sigma, \mathcal{N}}$ is completely positive, trace non-increasing, and unique on the support of $\mathcal{N}(\sigma)$ [28-31]. In the case that the Hilbert spaces $A$ and $B$ are finite-dimensional, then, on the support of $\mathcal{N}(\sigma)$, the Petz recovery map takes the form

$$
\mathcal{P}_{\sigma, \mathcal{N}}: X_{B} \mapsto \sigma^{\frac{1}{2}} \mathcal{N}^{\dagger}\left(\mathcal{N}(\sigma)^{-\frac{1}{2}} X_{B} \mathcal{N}(\sigma)^{-\frac{1}{2}}\right) \sigma^{\frac{1}{2}}
$$

(Following the standard convention, $\sigma^{-1}$ is defined to be the inverse of $\sigma$ on its support.)

\footnotetext{
${ }^{2}$ Note that the adjoint $\mathcal{N}^{\dagger}$ of $\mathcal{N}$ is defined as the unique linear map satisfying $\left\langle a, \mathcal{N}^{\dagger}(b)\right\rangle=$ $\langle\mathcal{N}(a), b\rangle$ for all $a \in \mathrm{TC}(A)$ and $b \in \mathrm{L}(B)$, where $\left\langle a_{1}, a_{2}\right\rangle:=\operatorname{tr}\left(a_{1}^{\dagger} a_{2}\right)$ is the Frobenius inner product.
} 
The concept of sufficient statistics can be made robust. For $\varepsilon \in[0,1]$, a quantum channel $\mathcal{N} \in \operatorname{TPCP}(A, B)$ is $\varepsilon$-sufficient with respect to $\mathrm{Q}(A)$ if there exists a recovery map $\mathcal{R}_{\varepsilon} \in \operatorname{TPCP}(B, A)$ such that [15]

$$
\frac{1}{2}\left\|\rho-\left(\mathcal{R}_{\varepsilon} \circ \mathcal{N}\right)(\rho)\right\|_{1} \leq \varepsilon \text { for all } \rho \in \mathrm{Q}(A) .
$$

Together with the case $\varepsilon=0$ discussed above and ideas from [44], this motivates the question if there exists a stronger version of the data processing inequality. More precisely, one asks for the possibility of adding a nonnegative term to the right-hand side of (3) that indicates how well $\rho$ can be recovered from $\mathcal{N}(\rho)$. Such a relation would serve as an alternative characterization of approximate sufficient statistics.

An inequality that is closely related to the monotonicity of the relative entropy is the strong subadditivity of quantum entropy [20,21], which ensures that for any tripartite state $\rho_{A B C} \in \mathrm{S}(A \otimes B \otimes C)$ the conditional mutual information is nonnegative, i.e., $I(A: C \mid B)_{\rho}:=H(A B)_{\rho}+H(B C)_{\rho}-H(A B C)_{\rho}-$ $H(B)_{\rho} \geq 0$, where $H(A)_{\rho}:=-\operatorname{tr}\left(\rho_{A} \log \rho_{A}\right)$ denotes the von Neumann entropy. ${ }^{3}$ This inequality has been strengthened recently with a remainder term in the form of a distance to the closest recovered state. It was shown in [8], that for any density operator $\rho_{A B C}$, there exists $\mathcal{R}_{B \rightarrow B C} \in \operatorname{TPCP}(B, B \otimes C)$ (the recovery map) such that

$$
I(A: C \mid B)_{\rho} \geq-2 \log F\left(\rho_{A B C}, \mathcal{R}_{B \rightarrow B C}\left(\rho_{A B}\right)\right),
$$

where the fidelity of $\rho$ and $\sigma$ is defined by [40]

$$
F(\rho, \sigma):=\|\sqrt{\rho} \sqrt{\sigma}\|_{1} .
$$

If $A, B$, and $C$ are finite-dimensional Hilbert spaces, on the support of $\rho_{B}$, $\mathcal{R}_{B \rightarrow B C}$ can be taken as a rotated Petz recovery map, i.e., a trace-preserving completely positive map of the form

$$
X_{B} \mapsto V_{B C} \rho_{B C}^{\frac{1}{2}}\left(\rho_{B}^{-\frac{1}{2}} U_{B} X_{B} U_{B}^{\dagger} \rho_{B}^{-\frac{1}{2}} \otimes \operatorname{id}_{C}\right) \rho_{B C}^{\frac{1}{2}} V_{B C}^{\dagger},
$$

where $V_{B C}$ and $U_{B}$ are unitaries acting on $B \otimes C$ and $B$, respectively.

The result of [8], whose proof is based on de Finetti-type arguments and properties of Rényi entropies, has been extended and generalized in various ways. In [6], based on the quantum state redistribution protocol [7] and de Finetti-type arguments, it was shown that the fidelity term can be replaced by a measured relative entropy $D_{\mathbb{M}}$, which is never smaller than the fidelity term, i.e.,

$$
I(A: C \mid B)_{\rho} \geq D_{\mathbb{M}}\left(\rho_{A B C} \| \mathcal{R}_{B \rightarrow B C}\left(\rho_{A B}\right)\right) \geq-2 \log F\left(\rho_{A B C}, \mathcal{R}_{B \rightarrow B C}\left(\rho_{A B}\right)\right) .
$$

\footnotetext{
3 The definition of conditional mutual information we have given is for finite-dimensional spaces and can be extended to the infinite-dimensional case [36].
} 
The measured relative entropy is defined as the supremum of the relative entropy with measured inputs over all positive operator-valued measures (POVMs) $\mathcal{M}=\left\{M_{x}\right\}$, i.e.,

$$
\begin{aligned}
& D_{\mathbb{M}}(\rho \| \sigma):=\sup \{D(\mathcal{M}(\rho) \| \mathcal{M}(\sigma)): \\
&\left.\qquad \mathcal{M}(\rho)=\sum_{x} \operatorname{tr}\left(\rho M_{x}\right)|x\rangle\langle x| \text { with } \sum_{x} M_{x}=\mathrm{id}\right\},
\end{aligned}
$$

where $\{|x\rangle\}_{x}$ is a finite set of orthonormal vectors. We note that the tighter bound from [6] came at the cost of losing all information about the structure of the recovery map. In [38], it was shown that there exists a recovery map both satisfying (10) and possessing a universality property, in the sense that it only depends on the marginal $\rho_{B C}$. Furthermore, for a linearized version of (7) it was shown that the recovery map has the form of a rotated Petz recovery map with commuting unitaries, i.e., a recovery map of the form in (9) where $V_{B C}$ and $U_{B}$ commute with $\rho_{B C}$ and $\rho_{B}$, respectively.

In view of approximate sufficiency of quantum channels discussed above, it would be helpful to have a generalization of (7) in terms of relative entropies. This has been established in [42] with a proof technique based on the notion of a Rényi generalization of a relative entropy difference [35] and Hadamard's three-line theorem. It was shown that for any two states $\rho$ and $\sigma$ acting on finite-dimensional Hilbert spaces with $\operatorname{supp}(\rho) \subseteq \operatorname{supp}(\sigma)$ and any channel $\mathcal{N}$ there exists a recovery map $\mathcal{R}$ such that $(\mathcal{R} \circ \mathcal{N})(\sigma)=\sigma$ and

$$
D(\rho \| \sigma)-D(\mathcal{N}(\rho) \| \mathcal{N}(\sigma)) \geq-2 \log F(\rho,(\mathcal{R} \circ \mathcal{N})(\rho)) .
$$

Furthermore, the recovery map was shown to be a rotated Petz recovery map with unitaries $U$ and $V$ in the algebra generated by $\sigma$ and $\mathcal{N}(\sigma)$, respectively. Very recently, another different proof technique was found [39], based on the concavity and monotonicity of the operator logarithm, which shows that there exists a recovery map $\mathcal{R}$ such that

$$
\begin{aligned}
D(\rho \| \sigma)-D(\mathcal{N}(\rho) \| \mathcal{N}(\sigma)) & \geq D_{\mathbb{M}}(\rho \|(\mathcal{R} \circ \mathcal{N})(\rho)) \\
& \geq-2 \log F(\rho,(\mathcal{R} \circ \mathcal{N})(\rho)) .
\end{aligned}
$$

The recovery map was shown to be a convex combination of rotated Petz recovery maps with unitaries $U$ and $V$ in the algebra generated by $\sigma$ and $\mathcal{N}(\sigma)$, respectively, and therefore satisfies $(\mathcal{R} \circ \mathcal{N})(\sigma)=\sigma$.

Neither in [42] nor in [39] was the recovery map satisfying (12) and (13), respectively, shown to be universal, in the sense that it could be taken independent of $\rho$. We note that by the Fuchs--van de Graaf inequality [9] the fidelity can be transferred into a trace distance term such that (12) and (14) provide alternative characterizations for approximate sufficient statistics.

Result. We show that for any nonnegative operator $\sigma$ and for any channel $\mathcal{N}$ there exists an explicit and universal recovery map $\mathcal{R}_{\sigma, \mathcal{N}}$ such that

$$
D(\rho \| \sigma) \geq D(\mathcal{N}(\rho) \| \mathcal{N}(\sigma))-2 \log F\left(\rho,\left(\mathcal{R}_{\sigma, \mathcal{N}} \circ \mathcal{N}\right)(\rho)\right)
$$


for all density operators $\rho$ such that $\operatorname{supp}(\rho) \subseteq \operatorname{supp}(\sigma)$. A consequence of the universality of the recovery map $\mathcal{R}_{\sigma, \mathcal{N}}$ is that $\left(\mathcal{R}_{\sigma, \mathcal{N}} \circ \mathcal{N}\right)(\sigma)=\sigma$. We note that $\rho$ and $\sigma$ are defined on separable Hilbert spaces. We refer to Theorem 2.1 and Remark 2.2 for a more precise statement, and we note here that the result stated in Theorem 2.1 is strictly stronger than (15).

History of the Problem. In 1973, the strong subadditivity of quantum entropy $[20,21]$ was proven. It ensures that the conditional mutual information of any tripartite state is nonnegative. Two years later the data processing inequality, or monotonicity of the quantum relative entropy under trace-preserving completely positive maps was proven [24,41]. This entropy inequality states that the quantum relative entropy cannot increase after applying a quantum channel to its arguments. Since then it has been realized that this fundamental theorem has numerous applications in quantum physics, and as a consequence, it was natural to ask if it would be possible to strengthen the result. This, however, turned out to be challenging. More recently, several conjectures regarding an improved data processing inequality have been put forward. (See [44] for one of these conjectures). In this paper, we prove (15) with a recovery map that satisfies all the properties that have been conjectured to hold (see, e.g., [44]).

\section{Main Results}

Let $\mathrm{P}(A)$ denote the set of nonnegative trace-class operators acting on a Hilbert space $A$. For any $\sigma \in \mathrm{P}(A)$, we define

$$
\mathrm{S}_{\sigma}(A):=\{\rho \in \mathrm{S}(A): \operatorname{supp}(\rho) \subseteq \operatorname{supp}(\sigma)\} .
$$

Theorem 2.1. Let $A$ and $B$ be separable Hilbert spaces. For any $\sigma \in \mathrm{P}(A)$, any $\rho \in \mathrm{S}_{\sigma}(A)$ and any $\mathcal{N} \in \operatorname{TPCP}(A, B)$, we have

$$
D(\rho \| \sigma) \geq D(\mathcal{N}(\rho) \| \mathcal{N}(\sigma))-2 \int_{\mathbb{R}} \mathrm{d} t \beta_{0}(t) \log F\left(\rho,\left(\mathcal{R}_{\sigma, \mathcal{N}}^{\frac{t}{2}} \circ \mathcal{N}\right)(\rho)\right),
$$

where the relative entropy and fidelity are defined in (2) and (8), respectively. The recovery map is given by

$$
\mathcal{R}_{\sigma, \mathcal{N}}^{t}: X_{B} \mapsto \sigma^{-\mathrm{i} t} \mathcal{P}_{\sigma, \mathcal{N}}\left(\mathcal{N}(\sigma)^{\mathrm{i} t} X_{B} \mathcal{N}(\sigma)^{-\mathrm{i} t}\right) \sigma^{\mathrm{i} t}
$$

and $\beta_{0}$ is a probability density function on $\mathbb{R}$ defined by

$$
\beta_{0}(t):=\frac{\pi}{2}(\cosh (\pi t)+1)^{-1} .
$$

The map $\mathcal{P}_{\sigma, \mathcal{N}}$ is the Petz recovery map, defined as the adjoint of the unique linear map $\mathcal{P}_{\sigma, \mathcal{N}}^{\dagger}$ satisfying (4) with domain $\mathrm{L}(\operatorname{supp}(\mathcal{N}(\sigma)))$ and range $\mathrm{L}(\operatorname{supp}(\sigma))$. If $A$ and $B$ are finite-dimensional, this unique linear map $\mathcal{P}_{\sigma, \mathcal{N}}$ is given by (5).

Remark 2.2. Using the concavity of the logarithm and the fidelity [27, Exercise 9.20], Theorem 2.1 can be simplified to

$$
D(\rho \| \sigma) \geq D(\mathcal{N}(\rho) \| \mathcal{N}(\sigma))-2 \log F\left(\rho,\left(\mathcal{R}_{\sigma, \mathcal{N}} \circ \mathcal{N}\right)(\rho)\right),
$$




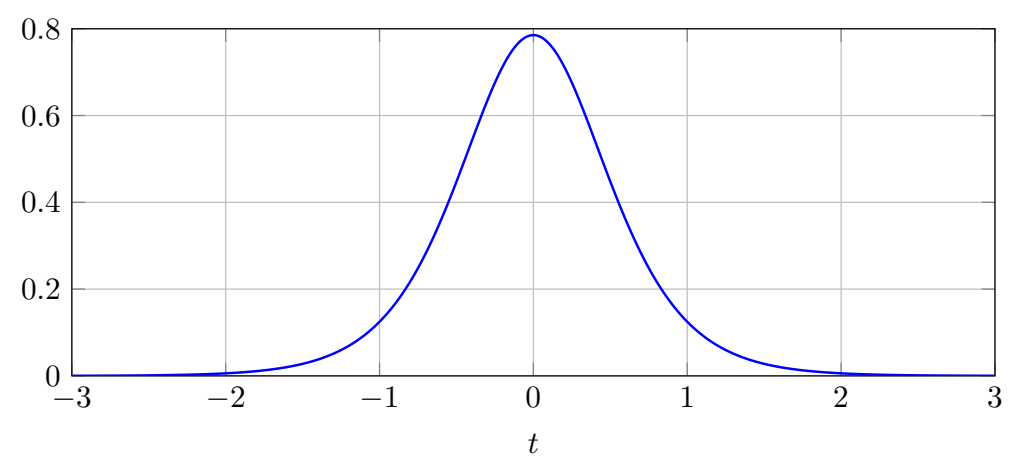

Figure 1. This plot depicts the probability density $\beta_{0}$ defined in (19) as a function of $t \in \mathbb{R}$. We see that it is peaked around $t=0$ which corresponds to the Petz recovery map, i.e., $\mathcal{R}_{\sigma, \mathcal{N}}^{t=0}=\mathcal{P}_{\sigma, \mathcal{N}}$.

where

$$
\mathcal{R}_{\sigma, \mathcal{N}}(\cdot):=\int_{\mathbb{R}} \mathrm{d} t \beta_{0}(t) \mathcal{R}_{\sigma, \mathcal{N}}^{\frac{t}{2}}(\cdot)
$$

on the support of $\mathcal{N}(\sigma)$ with $\mathcal{R}_{\sigma, \mathcal{N}}^{t}$ and $\beta_{0}$ defined in Theorem 2.1.

Figure 1 depicts the probability density $\beta_{0}$ as a function of $t \in \mathbb{R}$. We note that the recovery map $\mathcal{R}_{\sigma, \mathcal{N}}$ that satisfies $(20)$ can be chosen such that it projects everything outside of the support of $\mathcal{N}(\sigma)$ to zero.

Remark 2.3. Inequality (17) together with the fact that the mapping $t \mapsto \mathcal{R}_{\sigma, \mathcal{N}}^{t}$ is continuous implies that for any $\sigma \in \mathrm{P}(A), \rho \in \mathrm{S}_{\sigma}(A)$ and $\mathcal{N} \in \operatorname{TPCP}(A, B)$ such that $D(\rho \| \sigma)=D(\mathcal{N}(\rho) \| \mathcal{N}(\sigma))$ we have $\left(\mathcal{R}_{\sigma, \mathcal{N}}^{t} \circ \mathcal{N}\right)(\rho)=\rho$ and $\left(\mathcal{R}_{\sigma, \mathcal{N}}^{t} \circ\right.$ $\mathcal{N})(\sigma)=\sigma$ for all $t \in \mathbb{R}$ with $\mathcal{R}_{\sigma, \mathcal{N}}^{t}(\cdot)$ defined in (18). This follows because $F(\omega, \tau) \in[0,1]$ and $F(\omega, \tau)=1$ if and only if $\omega=\tau$ for density operators $\omega$ and $\tau$.

Remark 2.4 (Functoriality properties). The recovery map $\mathcal{R}_{\sigma, \mathcal{N}}$ stated in Remark 2.2 satisfies apart from (20) several desirable "functoriality" properties. Some of them have been stated in $[19,42,44]$.

1. Universality. The recovery map does not depend on $\rho$. This follows directly from Remark 2.2.

2. Perfect reconstruction of $\sigma$ from $\mathcal{N}(\sigma)$. The recovery map satisfies $\left(\mathcal{R}_{\sigma, \mathcal{N}} \circ \mathcal{N}\right)(\sigma)=\sigma$. This is clear from the fact that any rotated Petz map of the form in (18) perfectly recovers $\sigma$ [42], and thus so does any convex combination of these maps. Alternatively, as the recovery map predicted by Remark 2.2 that satisfies (20) is universal, the assertion follows by choosing $\rho=\sigma / \operatorname{tr}(\sigma)$.

\footnotetext{
4 The integral in (21) can be understood as a Riemann sum with respect to weak convergence since we have a weakly continuous family of maps.
} 
3. Normalization. In case $\mathcal{N}=\mathcal{I}$, where $\mathcal{I}$ denotes the identity map, we have $\mathcal{R}_{\sigma, \mathcal{N}}(\cdot)=\Pi_{\sigma}(\cdot) \Pi_{\sigma}$, where $\Pi_{\sigma}$ denotes a projector onto the support of $\sigma$. Thus, if $\sigma$ is faithful, the recovery map is equal to the identity channel. This follows directly by [42, Section 4.1] and by definition of the recovery $\operatorname{map} \mathcal{R}_{\sigma, \mathcal{N}}(\cdot)$.

4. Stabilization. For any $\sigma \in \mathrm{P}(A)$, any $\mathcal{N} \in \operatorname{TPCP}(A, B)$, any reference sys-

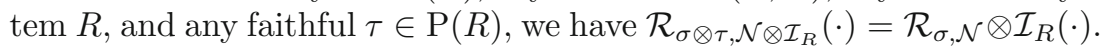
This follows by combining [42, Section 4.2] together with the normalization property discussed above.

We note that by following [42, Sections 4.2 and 4.3] it can be shown that the recovery map $\mathcal{R}_{\sigma, \mathcal{N}}$ fulfills some parallel and serial composition rules.

The proof of Theorem 2.1 consists of two parts. We first prove the statement for finite-dimensional Hilbert spaces $A$ and $B$ by employing a strengthened version of Hadamard's three-line theorem that is due to Hirschman [13]. By an approximation argument we show that the result remains valid for separable Hilbert spaces.

\section{Proof of Theorem 2.1}

\section{Step 1: Proof for Finite-Dimensional Hilbert Spaces}

In this step, we assume that the Hilbert spaces $A$ and $B$ are finite-dimensional. Our proof of (17) is similar to the approach taken in [42]. There are two main ingredients: a Rényi generalization of a relative entropy difference [35] and Hirschman's improvement of the Hadamard three-line theorem [13]. We begin by recalling these two ingredients and then proceed to a proof of (17).

For any $L \in \mathrm{L}(A)$ the Schatten $p$-norm is defined as

$$
\|L\|_{p}:=\left(\operatorname{tr}\left(|L|^{p}\right)\right)^{\frac{1}{p}} \quad \text { for } \quad p \in[1, \infty),
$$

where $|L|:=\sqrt{L^{\dagger} L}$. A Rényi generalization of a relative entropy difference ${ }^{5}$ is defined as [35]

$$
\widetilde{\Delta}_{\alpha}(\rho, \sigma, \mathcal{N}):=\frac{2 \alpha}{\alpha-1} \log \left\|\left([\mathcal{N}(\rho)]^{\frac{1-\alpha}{2 \alpha}}[\mathcal{N}(\sigma)]^{\frac{\alpha-1}{2 \alpha}} \otimes \operatorname{id}_{E}\right) U_{A \rightarrow B E} \sigma^{\frac{1-\alpha}{2 \alpha}} \rho^{\frac{1}{2}}\right\|_{2 \alpha},
$$

where $\alpha \in(0,1) \cup(1, \infty)$, and $U_{A \rightarrow B E}$ is an isometric extension of the channel $\mathcal{N}$. That is, $U_{A \rightarrow B E}$ is a linear isometry satisfying $\operatorname{tr}_{E}\left(U_{A \rightarrow B E}(\cdot) U_{A \rightarrow B E}^{\dagger}\right)=$ $\mathcal{N}(\cdot)$ and $U_{A \rightarrow B E}^{\dagger} U_{A \rightarrow B E}=\mathrm{id}_{A}$. All isometric extensions of a channel are related by an isometry acting on the environment system $E$, so that the definition in (23) is invariant under any such choice. Recall also that the adjoint $\mathcal{N}^{\dagger}$ of a channel is given in terms of an isometric extension $U$ as $\mathcal{N}^{\dagger}(\cdot)=U^{\dagger}\left((\cdot) \otimes \operatorname{id}_{E}\right) U$. The following lemma was established in [35] for the case in which $\rho, \sigma, \mathcal{N}(\rho)$, and $\mathcal{N}(\sigma)$ are positive definite and was later extended in the appendix of [42] to hold for the case in which $\rho \in \mathrm{S}_{\sigma}(A)$ :

\footnotetext{
5 The explanation in which sense this term is a relative entropy difference is given in (24).
} 
Lemma 3.1. ([35,42]) Let $A$ and $B$ be finite-dimensional Hilbert spaces. The following limit holds for $\sigma \in \mathrm{P}(A), \rho \in \mathrm{S}_{\sigma}(A)$, and $\mathcal{N} \in \operatorname{TPCP}(A, B)$ :

$$
\lim _{\alpha \rightarrow 1} \widetilde{\Delta}_{\alpha}(\rho, \sigma, \mathcal{N})=D(\rho \| \sigma)-D(\mathcal{N}(\rho) \| \mathcal{N}(\sigma))
$$

For $\alpha=\frac{1}{2}$, observe that

$$
\begin{aligned}
\widetilde{\Delta}_{\frac{1}{2}}(\rho, \sigma, \mathcal{N}) & =-2 \log \left\|\left([\mathcal{N}(\rho)]^{\frac{1}{2}}[\mathcal{N}(\sigma)]^{-\frac{1}{2}} \otimes \operatorname{id}_{E}\right) U_{A \rightarrow B E} \sigma^{\frac{1}{2}} \rho^{\frac{1}{2}}\right\|_{1} \\
& =-2 \log F\left(\rho, \mathcal{P}_{\sigma, \mathcal{N}} \circ \mathcal{N}(\rho)\right)
\end{aligned}
$$

where $\mathcal{P}_{\sigma, \mathcal{N}}$ denotes the Petz recovery map defined in (5).

The following lemma is based on Hirschman's improvement of the Hadamard three-line theorem [13], and for completeness, we provide a proof in "Appendix A."

Lemma 3.2. Let $S:=\{z \in \mathbb{C}: 0 \leq \operatorname{Re}\{z\} \leq 1\}$ and let $G: S \rightarrow L(\mathcal{H})$ be a bounded map that is holomorphic on the interior of $S$ and continuous on the boundary. Let $\theta \in(0,1)$ and define $p_{\theta}$ by

$$
\frac{1}{p_{\theta}}=\frac{1-\theta}{p_{0}}+\frac{\theta}{p_{1}}
$$

where $p_{0}, p_{1} \in[1, \infty]$. Then, the following bound holds

$$
\log \left(\|G(\theta)\|_{p_{\theta}}\right) \leq \int_{\mathbb{R}} \mathrm{d} t\left(\alpha_{\theta}(t) \log \left(\|G(\mathrm{i} t)\|_{p_{0}}^{1-\theta}\right)+\beta_{\theta}(t) \log \left(\|G(1+\mathrm{i} t)\|_{p_{1}}^{\theta}\right)\right),
$$

where $\alpha_{\theta}(t)$ and $\beta_{\theta}(t)$ are defined by

$$
\alpha_{\theta}(t):=\frac{\sin (\pi \theta)}{2(1-\theta)(\cosh (\pi t)-\cos (\pi \theta))} \quad \text { and } \quad \beta_{\theta}(t):=\frac{\sin (\pi \theta)}{2 \theta(\cosh (\pi t)+\cos (\pi \theta))} .
$$

Remark 3.3. Fix $\theta \in(0,1)$. Observe that $\alpha_{\theta}(t), \beta_{\theta}(t) \geq 0$ for all $t \in \mathbb{R}$ and we have

$$
\int_{\mathbb{R}} \mathrm{d} t \alpha_{\theta}(t)=\int_{\mathbb{R}} \mathrm{d} t \beta_{\theta}(t)=1,
$$

(see, e.g., [10, Exercise 1.3.8]) so that $\alpha_{\theta}(t)$ and $\beta_{\theta}(t)$ can be interpreted as probability density functions. Furthermore, the following limit holds

$$
\lim _{\theta \searrow 0} \beta_{\theta}(t)=\frac{\pi}{2(\cosh (\pi t)+1)}=\beta_{0}(t)
$$

where $\beta_{0}$ is also a probability density function on $\mathbb{R}$.

We can now readily establish the desired result in (17) for the finitedimensional case. In what follows, we abbreviate the isometric extension $U_{A \rightarrow B E}$ of the channel $\mathcal{N}$ as $U$. Pick

$$
G(z):=\left([\mathcal{N}(\rho)]^{\frac{z}{2}}[\mathcal{N}(\sigma)]^{-\frac{z}{2}} \otimes \operatorname{id}_{E}\right) U \sigma^{\frac{z}{2}} \rho^{\frac{1}{2}},
$$


$p_{0}=2, p_{1}=1$, and $\theta \in(0,1)$, which fixes $p_{\theta}=\frac{2}{1+\theta}$. The operator-valued function $G(z)$ satisfies the conditions needed to apply Lemma 3.2. For the choices above, we find that

$$
\|G(\theta)\|_{\frac{2}{1+\theta}}=\left\|\left([\mathcal{N}(\rho)]^{\frac{\theta}{2}}[\mathcal{N}(\sigma)]^{-\frac{\theta}{2}} \otimes \operatorname{id}_{E}\right) U \sigma^{\frac{\theta}{2}} \rho^{\frac{1}{2}}\right\|_{\frac{2}{1+\theta}},
$$

and

$$
\|G(\mathrm{i} t)\|_{2}=\left\|\left([\mathcal{N}(\rho)]^{\frac{\mathrm{i} t}{2}}[\mathcal{N}(\sigma)]^{-\frac{\mathrm{i} t}{2}} \otimes \mathrm{id}_{E}\right) U \sigma^{\mathrm{i} t} \rho^{\frac{1}{2}}\right\|_{2} \leq\left\|\rho^{\frac{1}{2}}\right\|_{2}=1,
$$

as well as

$$
\begin{aligned}
\|G(1+\mathrm{i} t)\|_{1} & =\left\|\left([\mathcal{N}(\rho)]^{\frac{1+\mathrm{i} t}{2}}[\mathcal{N}(\sigma)]^{-\frac{1+\mathrm{i} t}{2}} \otimes \mathrm{id}_{E}\right) U \sigma^{\frac{1+\mathrm{i} t}{2}} \rho^{\frac{1}{2}}\right\|_{1} \\
& =\left\|\left([\mathcal{N}(\rho)]^{\frac{\mathrm{i} t}{2}}[\mathcal{N}(\rho)]^{\frac{1}{2}}[\mathcal{N}(\sigma)]^{-\frac{\mathrm{i} t}{2}}[\mathcal{N}(\sigma)]^{-\frac{1}{2}} \otimes \mathrm{id}_{E}\right) U \sigma^{\frac{1}{2}} \sigma^{\frac{\mathrm{i} t}{2}} \rho^{\frac{1}{2}}\right\|_{1} \\
& =\left\|\left([\mathcal{N}(\rho)]^{\frac{1}{2}}[\mathcal{N}(\sigma)]^{-\frac{\mathrm{i} t}{2}}[\mathcal{N}(\sigma)]^{-\frac{1}{2}} \otimes \mathrm{id}_{E}\right) U \sigma^{\frac{1}{2}} \sigma^{\frac{\mathrm{i} t}{2}} \rho^{\frac{1}{2}}\right\|_{1} \\
& =F\left(\rho,\left(\mathcal{R}_{\sigma, \mathcal{N}}^{\frac{t}{2}} \circ \mathcal{N}\right)(\rho)\right) .
\end{aligned}
$$

Then, we can apply the fact that $\|G(\mathrm{it})\|_{2} \leq 1$ and (27) to conclude that the following bound holds for all $\theta \in(0,1)$

$$
\begin{aligned}
\log \|\left([\mathcal{N}(\rho)]^{\frac{\theta}{2}}[\mathcal{N}(\sigma)]^{-\frac{\theta}{2}} \otimes \operatorname{id}_{E}\right) U & \sigma^{\frac{\theta}{2}} \rho^{\frac{1}{2}} \|_{\frac{2}{1+\theta}} \\
& \leq \int_{\mathbb{R}} \mathrm{d} t \beta_{\theta}(t) \log \left(F\left(\rho,\left(\mathcal{R}_{\sigma, \mathcal{N}}^{\frac{t}{2}} \circ \mathcal{N}\right)(\rho)\right)^{\theta}\right),
\end{aligned}
$$

which implies

$$
\begin{aligned}
-\frac{2}{\theta} \log \|\left([\mathcal{N}(\rho)]^{\frac{\theta}{2}}[\mathcal{N}(\sigma)]^{-\frac{\theta}{2}} \otimes \operatorname{id}_{E}\right) & U \sigma^{\frac{\theta}{2}} \rho^{\frac{1}{2}} \|_{\frac{2}{1+\theta}} \\
& \geq-2 \int_{\mathbb{R}} \mathrm{d} t \beta_{\theta}(t) \log F\left(\rho,\left(\mathcal{R}_{\sigma, \mathcal{N}}^{\frac{t}{2}} \circ \mathcal{N}\right)(\rho)\right) .
\end{aligned}
$$

Letting $\theta=\frac{1-\alpha}{\alpha}$, we see that this is the same as

$$
\widetilde{\Delta}_{\alpha}(\rho, \sigma, \mathcal{N}) \geq-2 \int_{\mathbb{R}} \mathrm{d} t \beta_{\frac{1-\alpha}{\alpha}}(t) \log F\left(\rho,\left(\mathcal{R}_{\sigma, \mathcal{N}}^{\frac{t}{2}} \circ \mathcal{N}\right)(\rho)\right) .
$$

Since the inequality in (36) holds for all $\theta \in(0,1)$ and thus (37) holds for all $\alpha \in\left(\frac{1}{2}, 1\right)$, we can take the limit as $\alpha \nearrow 1$ and apply $(24),(30)$, and the dominated convergence theorem to conclude that (17) holds.

Remark 3.4. If $A$ and $B$ are finite-dimensional Hilbert spaces the statement in Remark 2.2 can be slightly generalized. For any $\sigma \geq 0$ and any trace nonincreasing completely positive map $\mathcal{N}$ with Kraus operators $\left\{N_{i}\right\}$ such that $0 \neq \sum_{i} N_{i}^{\dagger} N_{i} \leq$ id the recovery map $\mathcal{R}_{\sigma, \mathcal{N}}$ defined in Remark 2.2 satisfies (20) for all subnormalized density operators $\rho \geq 0$ with $0<\operatorname{tr}(\rho) \leq 1$ such that $\operatorname{supp}(\rho) \subseteq \operatorname{supp}(\sigma)$. This follows by the same argument given in Step 1 
by using $U_{A \rightarrow B E}=\sum_{i} N_{i} \otimes|i\rangle_{E}$ as an extension of the trace non-increasing and completely positive map $\mathcal{N}$.

\section{Step 2: Extension to Infinite Dimensions}

In this step, the Hilbert spaces $A$ and $B$ are assumed to be separable (not necessarily finite-dimensional). We will show how to lift Theorem 2.1 for finite-dimensional Hilbert spaces, such that it can apply to states $\rho$ and $\sigma$ and a channel $\mathcal{N}$ associated with separable Hilbert spaces. This is accomplished via a limiting argument where we consider projected sequences that are finite-dimensional and therefore satisfy the desired inequality. In the limit, we then obtain the statement for separable Hilbert spaces. This is a rather standard approach for generalizing statements proven for finite-dimensional Hilbert spaces to separable Hilbert spaces.

Let $\left\{\Pi_{A}^{a}\right\}_{a \in \mathbb{N}}$ and $\left\{\Pi_{B}^{b}\right\}_{b \in \mathbb{N}}$ be sequences of finite-rank projectors on $A$ and $B$, respectively, that converge to $\mathrm{id}_{A}$ and $\mathrm{id}_{B}$, respectively, with respect to the weak operator topology, meaning that

$$
\lim _{a \rightarrow \infty}\left\langle\psi\left|\Pi_{A}^{a}\right| \phi\right\rangle=\langle\psi \mid \phi\rangle
$$

for all vectors $|\phi\rangle,|\psi\rangle \in A$ (similarly for $\Pi_{B}^{b} \rightarrow \mathrm{id}_{B}$ ). For $\sigma \in \mathrm{P}(A)$ and $\rho \in \mathrm{S}_{\sigma}(A)$, we consider projected versions

$$
\sigma^{a}:=\Pi_{A}^{a} \sigma \Pi_{A}^{a} \quad \text { and } \quad \rho^{a}:=\Pi_{A}^{a} \rho \Pi_{A}^{a} .
$$

We note that the sequences $\left\{\rho^{a}\right\}_{a \in \mathbb{N}}$ and $\left\{\sigma^{a}\right\}_{a \in \mathbb{N}}$ converge to $\rho$ and $\sigma$, respectively, in the trace norm (see, e.g., [11] or Lemma 11.1 of [14]). Let $\mathrm{S}^{a}$ be the set of nonnegative operators that is generated by (39) for all $\rho \in \mathrm{S}(A)$. For any $\mathcal{N} \in \operatorname{TPCP}(A, B)$, we define its analogue with a projection at the input and output as

$$
\mathcal{N}^{a, b}(\cdot):=\Pi_{B}^{b} \mathcal{N}\left(\Pi_{A}^{a}(\cdot) \Pi_{A}^{a}\right) \Pi_{B}^{b} .
$$

Note that by combining Grümm's theorem [37, Theorem 2.19] with the boundedness of $\mathcal{N}$ in the trace norm implies that $\mathcal{N}^{a, b}$ converges to $\mathcal{N}$ in the strong operator topology, i.e.,

$$
\lim _{a, b \rightarrow \infty}\left\|\mathcal{N}^{a, b}(\omega)-\mathcal{N}(\omega)\right\|_{1}=0
$$

for all $\omega \in \operatorname{TC}(A)$.

We start by proving two lemmas that show how the difference of relative entropies and the fidelity, respectively, change when considering projected states.

Lemma 3.5. For any $\sigma \in \mathrm{P}(A)$, any $\rho \in \mathrm{S}_{\sigma}(A)$, and any $\mathcal{N} \in \operatorname{TPCP}(A, B)$, we have

$$
\lim _{a \rightarrow \infty} D\left(\rho^{a} \| \sigma^{a}\right)=D(\rho \| \sigma)
$$

and

$$
\liminf _{a \rightarrow \infty} \liminf _{b \rightarrow \infty} D\left(\mathcal{N}^{a, b}\left(\rho^{a}\right) \| \mathcal{N}^{a, b}\left(\sigma^{a}\right)\right) \geq D(\mathcal{N}(\rho) \| \mathcal{N}(\sigma))
$$


Proof. Recall that Lindblad defined a relative entropy (which slightly differs from our definition of the relative entropy) for two positive trace-class operators $\tau$ and $\omega$ as

$$
\bar{D}(\tau \| \omega):=\sum_{j}\langle j|(\tau \log \tau-\tau \log \omega+\omega-\tau)| j\rangle=D(\tau \| \omega)+\operatorname{tr}(\omega)-\operatorname{tr}(\tau),
$$

where $\{|j\rangle\}$ is a complete orthonormal set of eigenvectors of $\tau$ or $\omega$. By Lemma 3 in [23], we have

$$
\begin{aligned}
D\left(\rho^{a} \| \sigma^{a}\right) & =\bar{D}\left(\rho^{a} \| \sigma^{a}\right)+\operatorname{tr}\left(\rho^{a}\right)-\operatorname{tr}\left(\sigma^{a}\right) \\
& \leq \bar{D}(\rho \| \sigma)+\operatorname{tr}\left(\rho^{a}\right)-\operatorname{tr}\left(\sigma^{a}\right) \\
& =D(\rho \| \sigma)+\operatorname{tr}\left(\rho^{a}-\rho\right)+\operatorname{tr}\left(\sigma-\sigma^{a}\right) .
\end{aligned}
$$

Since the relative entropy is lower semicontinuous [14, Theorem 11.6], we obtain

$$
D(\rho \| \sigma) \leq \liminf _{a \rightarrow \infty} D\left(\rho^{a} \| \sigma^{a}\right) .
$$

Combining these shows that

$$
\lim _{a \rightarrow \infty} D\left(\rho^{a} \| \sigma^{a}\right)=D(\rho \| \sigma) .
$$

The lower semicontinuity of the relative entropy implies that

$$
\begin{aligned}
\liminf _{a \rightarrow \infty} \liminf _{b \rightarrow \infty} D\left(\mathcal{N}^{a, b}\left(\rho^{a}\right) \| \mathcal{N}^{a, b}\left(\sigma^{a}\right)\right) & \geq \liminf _{a \rightarrow \infty} D\left(\mathcal{N}\left(\rho^{a}\right) \| \mathcal{N}\left(\sigma^{a}\right)\right) \\
& \geq D(\mathcal{N}(\rho) \| \mathcal{N}(\sigma)) .
\end{aligned}
$$

This proves the assertion.

Lemma 3.6. The Petz recovery map $\mathcal{P}_{\sigma^{a}, \mathcal{N}^{a}, b}$ defined in (5) satisfies $\lim _{a \rightarrow \infty} \lim _{b \rightarrow \infty} \mathcal{P}_{\sigma^{a}, \mathcal{N} a, b}=\mathcal{P}_{\sigma, \mathcal{N}}$, where $\mathcal{P}_{\sigma, \mathcal{N}}$ is a recovery map that is defined as the (unique) linear map with domain $\operatorname{supp}(\mathcal{N}(\sigma))$ and range $\operatorname{supp}(\sigma)$ satisfying

$$
\left\langle a_{2}, \mathcal{N}^{\dagger}\left(a_{1}\right)\right\rangle_{\sigma}=\left\langle\mathcal{P}_{\sigma, \mathcal{N}}^{\dagger}\left(a_{2}\right), a_{1}\right\rangle_{\mathcal{N}(\sigma)} \quad \text { for all } \quad a_{1} \in \mathrm{L}(B), a_{2} \in \mathrm{L}(A),
$$

with the weighted inner product $\langle a, b\rangle_{\omega}:=\operatorname{tr}\left(a^{\dagger} \omega^{\frac{1}{2}} b \omega^{\frac{1}{2}}\right)$ and $\omega$ positive semidefinite and trace class.

Proof. We begin by outlining the proof. As shown by Petz [29-31] (see also [28, Chapter 8]), the map $\mathcal{P}_{\sigma^{a}, \mathcal{N}^{a, b}}$ defined in (5) is the unique linear map with domain $\operatorname{supp}\left(\mathcal{N}^{a, b}\left(\sigma^{a}\right)\right)$ and range $\operatorname{supp}\left(\sigma^{a}\right)$ satisfying

$$
\left\langle a_{2},\left(\mathcal{N}^{a, b}\right)^{\dagger}\left(a_{1}\right)\right\rangle_{\sigma^{a}}=\left\langle\mathcal{P}_{\sigma^{a}, \mathcal{N}^{a}, b}^{\dagger}\left(a_{2}\right), a_{1}\right\rangle_{\mathcal{N}^{a, b}\left(\sigma^{a}\right)} \quad \text { for all } \quad a_{1} \in \mathrm{L}(B), a_{2} \in \mathrm{L}(A),
$$

and the map $\mathcal{P}_{\sigma, \mathcal{N}}$ is the unique linear map with domain $\operatorname{supp}(\mathcal{N}(\sigma))$ and range $\operatorname{supp}(\sigma)$ satisfying

$$
\left\langle a_{2},(\mathcal{N})^{\dagger}\left(a_{1}\right)\right\rangle_{\sigma}=\left\langle\mathcal{P}_{\sigma, \mathcal{N}}^{\dagger}\left(a_{2}\right), a_{1}\right\rangle_{\mathcal{N}(\sigma)} \quad \text { for all } \quad a_{1} \in \mathrm{L}(B), a_{2} \in \mathrm{L}(A) .
$$


We will first show that

$$
\lim _{a \rightarrow \infty} \lim _{b \rightarrow \infty}\left\langle a_{2},\left(\mathcal{N}^{a, b}\right)^{\dagger}\left(a_{1}\right)\right\rangle_{\sigma^{a}}=\left\langle a_{2}, \mathcal{N}^{\dagger}\left(a_{1}\right)\right\rangle_{\sigma},
$$

for all $a_{1} \in \mathrm{L}(B), a_{2} \in \mathrm{L}(A)$, which by (53) and (54) implies that

$$
\lim _{a \rightarrow \infty} \lim _{b \rightarrow \infty}\left\langle\mathcal{P}_{\sigma^{a}, \mathcal{N}^{a, b}}^{\dagger}\left(a_{2}\right), a_{1}\right\rangle_{\mathcal{N}^{a, b}\left(\sigma^{a}\right)}=\left\langle\mathcal{P}_{\sigma, \mathcal{N}}^{\dagger}\left(a_{2}\right), a_{1}\right\rangle_{\mathcal{N}(\sigma)}
$$

for all $a_{1} \in \mathrm{L}(B), a_{2} \in \mathrm{L}(A)$. After showing that

$$
\lim _{a \rightarrow \infty} \lim _{b \rightarrow \infty}\left\langle\mathcal{P}_{\sigma^{a}, \mathcal{N}^{a}, b}^{\dagger}\left(a_{2}\right), a_{1}\right\rangle_{\mathcal{N}^{a, b}\left(\sigma^{a}\right)}=\lim _{a \rightarrow \infty} \lim _{b \rightarrow \infty}\left\langle\mathcal{P}_{\sigma^{a}, \mathcal{N}^{a}, b}^{\dagger}\left(a_{2}\right), a_{1}\right\rangle_{\mathcal{N}(\sigma)},
$$

we can conclude from (56) and (57) that

$$
\lim _{a \rightarrow \infty} \lim _{b \rightarrow \infty}\left\langle\mathcal{P}_{\sigma^{a}, \mathcal{N}^{a, b}}^{\dagger}\left(a_{2}\right), a_{1}\right\rangle_{\mathcal{N}(\sigma)}=\left\langle\mathcal{P}_{\sigma, \mathcal{N}}^{\dagger}\left(a_{2}\right), a_{1}\right\rangle_{\mathcal{N}(\sigma)} .
$$

From there, we argue that this implies the convergence $\lim _{a \rightarrow \infty} \lim _{b \rightarrow \infty}$ $\mathcal{P}_{\sigma^{a}, \mathcal{N} a, b}=\mathcal{P}_{\sigma, \mathcal{N}}$

Thus, we need to establish (55) and (57), and we begin by proving (55). Hölder's inequality implies that

$$
\begin{aligned}
\mid\left\langle a_{2},\right. & \left.\left(\mathcal{N}^{a, b}\right)^{\dagger}\left(a_{1}\right)\right\rangle_{\sigma^{a}}-\left\langle a_{2}, \mathcal{N}^{\dagger}\left(a_{1}\right)\right\rangle_{\sigma} \mid \\
= & \operatorname{tr}\left(\mathcal{N}^{a, b}\right)^{\dagger}\left(a_{1}\right)\left(\sigma^{a}\right)^{\frac{1}{2}} a_{2}^{\dagger}\left(\sigma^{a}\right)^{\frac{1}{2}}-\operatorname{tr} \mathcal{N}^{\dagger}\left(a_{1}\right) \sigma^{\frac{1}{2}} a_{2}^{\dagger} \sigma^{\frac{1}{2}} \mid \\
= & \operatorname{tr} a_{1}\left(\mathcal{N}^{a, b}\left(\left(\sigma^{a}\right)^{\frac{1}{2}} a_{2}^{\dagger}\left(\sigma^{a}\right)^{\frac{1}{2}}\right)-\mathcal{N}\left(\sigma^{\frac{1}{2}} a_{2}^{\dagger} \sigma^{\frac{1}{2}}\right)\right) \mid \\
\leq & \left\|a_{1}\right\|_{\infty}\left\|\mathcal{N}^{a, b}\left(\left(\sigma^{a}\right)^{\frac{1}{2}} a_{2}^{\dagger}\left(\sigma^{a}\right)^{\frac{1}{2}}\right)-\mathcal{N}\left(\sigma^{\frac{1}{2}} a_{2}^{\dagger} \sigma^{\frac{1}{2}}\right)\right\|_{1} \\
\leq & \left\|a_{1}\right\|_{\infty}\left\|\Pi_{B}^{b} \mathcal{N}\left(\left(\sigma^{a}\right)^{\frac{1}{2}} a_{2}^{\dagger}\left(\sigma^{a}\right)^{\frac{1}{2}}\right) \Pi_{B}^{b}-\mathcal{N}\left(\left(\sigma^{a}\right)^{\frac{1}{2}} a_{2}^{\dagger}\left(\sigma^{a}\right)^{\frac{1}{2}}\right)\right\|_{1} \\
& +\left\|a_{1}\right\|_{\infty}\left\|\mathcal{N}\left(\left(\sigma^{a}\right)^{\frac{1}{2}} a_{2}^{\dagger}\left(\sigma^{a}\right)^{\frac{1}{2}}\right)-\mathcal{N}\left(\sigma^{\frac{1}{2}} a_{2}^{\dagger} \sigma^{\frac{1}{2}}\right)\right\|_{1},
\end{aligned}
$$

where the final step uses the triangle inequality and that $\Pi_{A}^{a}\left(\sigma^{a}\right)^{\frac{1}{2}}=\left(\sigma^{a}\right)^{\frac{1}{2}}$. Grümm's theorem [37, Theorem 2.19] implies that for any fixed $a \in \mathbb{N}$ we have

$$
\lim _{b \rightarrow \infty}\left\|\Pi_{B}^{b} \mathcal{N}\left(\left(\sigma^{a}\right)^{\frac{1}{2}} a_{2}^{\dagger}\left(\sigma^{a}\right)^{\frac{1}{2}}\right) \Pi_{B}^{b}-\mathcal{N}\left(\left(\sigma^{a}\right)^{\frac{1}{2}} a_{2}^{\dagger}\left(\sigma^{a}\right)^{\frac{1}{2}}\right)\right\|_{1}=0
$$

and hence it remains to show that

$$
\lim _{a \rightarrow \infty}\left\|\mathcal{N}\left(\left(\sigma^{a}\right)^{\frac{1}{2}} a_{2}^{\dagger}\left(\sigma^{a}\right)^{\frac{1}{2}}\right)-\mathcal{N}\left(\sigma^{\frac{1}{2}} a_{2}^{\dagger} \sigma^{\frac{1}{2}}\right)\right\|_{1}=0
$$

To see this we first note that

$$
\left\|\mathcal{N}\left(\left(\sigma^{a}\right)^{\frac{1}{2}} a_{2}^{\dagger}\left(\sigma^{a}\right)^{\frac{1}{2}}\right)-\mathcal{N}\left(\sigma^{\frac{1}{2}} a_{2}^{\dagger} \sigma^{\frac{1}{2}}\right)\right\|_{1} \leq\|\mathcal{N}\|_{1 \rightarrow 1}\left\|\left(\sigma^{a}\right)^{\frac{1}{2}} a_{2}^{\dagger}\left(\sigma^{a}\right)^{\frac{1}{2}}-\sigma^{\frac{1}{2}} a_{2}^{\dagger} \sigma^{\frac{1}{2}}\right\|_{1}
$$

and by the triangle inequality

$$
\begin{aligned}
& \left\|\left(\sigma^{a}\right)^{\frac{1}{2}} a_{2}^{\dagger}\left(\sigma^{a}\right)^{\frac{1}{2}}-\sigma^{\frac{1}{2}} a_{2}^{\dagger} \sigma^{\frac{1}{2}}\right\|_{1} \\
& \quad \leq\left\|\left(\sigma^{a}\right)^{\frac{1}{2}} a_{2}^{\dagger}\left(\sigma^{a}\right)^{\frac{1}{2}}-\sigma^{\frac{1}{2}} a_{2}^{\dagger}\left(\sigma^{a}\right)^{\frac{1}{2}}\right\|_{1}+\left\|\sigma^{\frac{1}{2}} a_{2}^{\dagger}\left(\sigma^{a}\right)^{\frac{1}{2}}-\sigma^{\frac{1}{2}} a_{2}^{\dagger} \sigma^{\frac{1}{2}}\right\|_{1}
\end{aligned}
$$




$$
\begin{aligned}
& \leq\left\|\left(\sigma^{a}\right)^{\frac{1}{2}}-\sigma^{\frac{1}{2}}\right\|_{2}\left\|a_{2}\right\|_{\infty}\left\|\left(\sigma^{a}\right)^{\frac{1}{2}}\right\|_{2}+\left\|\sigma^{\frac{1}{2}}\right\|_{2}\left\|a_{2}\right\|_{\infty}\left\|\left(\sigma^{a}\right)^{\frac{1}{2}}-\sigma^{\frac{1}{2}}\right\|_{2} \\
& \leq 2\left\|a_{2}\right\|_{\infty}\left\|\left(\sigma^{a}\right)^{\frac{1}{2}}-\sigma^{\frac{1}{2}}\right\|_{2} \operatorname{tr}(\sigma) \\
& \leq 2\left\|a_{2}\right\|_{\infty}\left\|\sigma^{a}-\sigma\right\|_{1}^{\frac{1}{2}} \operatorname{tr}(\sigma),
\end{aligned}
$$

where second step follows by Hölder's inequality. The final step uses the Powers-Størmer inequality [32]. Inequality (69) implies (64) and thus proves $(55)$.

We now prove (57), by a reasoning that is very similar to the above. Hölder's inequality implies that

$$
\begin{aligned}
& \left|\left\langle\mathcal{P}_{\sigma^{a}, \mathcal{N}^{a}, b}^{\dagger}\left(a_{2}\right), a_{1}\right\rangle_{\mathcal{N}^{a, b}\left(\sigma^{a}\right)}-\left\langle\mathcal{P}_{\sigma^{a}, \mathcal{N}^{a, b}}^{\dagger}\left(a_{2}\right), a_{1}\right\rangle_{\mathcal{N}(\sigma)}\right| \\
& =\left|\operatorname{tr} a_{1}\left(\mathcal{N}^{a, b}\left(\sigma^{a}\right)^{\frac{1}{2}} \mathcal{P}_{\sigma^{a}, \mathcal{N}^{a, b}}^{\dagger}\left(a_{2}^{\dagger}\right) \mathcal{N}^{a, b}\left(\sigma^{a}\right)^{\frac{1}{2}}-\mathcal{N}(\sigma)^{\frac{1}{2}} \mathcal{P}_{\sigma^{a}, \mathcal{N}^{a}, b}^{\dagger}\left(a_{2}^{\dagger}\right) \mathcal{N}(\sigma)^{\frac{1}{2}}\right)\right| \\
& \leq\left\|a_{1}\right\|_{\infty}\left\|\mathcal{N}^{a, b}\left(\sigma^{a}\right)^{\frac{1}{2}} \mathcal{P}_{\sigma^{a}, \mathcal{N}^{a, b}}^{\dagger}\left(a_{2}^{\dagger}\right) \mathcal{N}^{a, b}\left(\sigma^{a}\right)^{\frac{1}{2}}-\mathcal{N}(\sigma)^{\frac{1}{2}} \mathcal{P}_{\sigma^{a}, \mathcal{N}^{a}, b}^{\dagger}\left(a_{2}^{\dagger}\right) \mathcal{N}(\sigma)^{\frac{1}{2}}\right\|_{1} .
\end{aligned}
$$

By the triangle inequality, we find

$$
\begin{aligned}
\left\|\mathcal{N}^{a, b}\left(\sigma^{a}\right)^{\frac{1}{2}} \mathcal{P}_{\sigma^{a}, \mathcal{N}^{a, b}}^{\dagger}\left(a_{2}^{\dagger}\right) \mathcal{N}^{a, b}\left(\sigma^{a}\right)^{\frac{1}{2}}-\mathcal{N}(\sigma)^{\frac{1}{2}} \mathcal{P}_{\sigma^{a}, \mathcal{N}^{a, b}}^{\dagger}\left(a_{2}^{\dagger}\right) \mathcal{N}(\sigma)^{\frac{1}{2}}\right\|_{1} \\
\leq\left\|\mathcal{N}^{a, b}\left(\sigma^{a}\right)^{\frac{1}{2}} \mathcal{P}_{\sigma^{a}, \mathcal{N} a, b}^{\dagger}\left(a_{2}^{\dagger}\right) \mathcal{N}^{a, b}\left(\sigma^{a}\right)^{\frac{1}{2}}-\mathcal{N}(\sigma)^{\frac{1}{2}} \mathcal{P}_{\sigma^{a}, \mathcal{N} a, b}^{\dagger}\left(a_{2}^{\dagger}\right) \mathcal{N}^{a, b}\left(\sigma^{a}\right)^{\frac{1}{2}}\right\|_{1} \\
\quad+\left\|\mathcal{N}(\sigma)^{\frac{1}{2}} \mathcal{P}_{\sigma^{a}, \mathcal{N}^{a, b}}^{\dagger}\left(a_{2}^{\dagger}\right) \mathcal{N}^{a, b}\left(\sigma^{a}\right)^{\frac{1}{2}}-\mathcal{N}(\sigma)^{\frac{1}{2}} \mathcal{P}_{\sigma^{a}, \mathcal{N} a, b}^{\dagger}\left(a_{2}^{\dagger}\right) \mathcal{N}(\sigma)^{\frac{1}{2}}\right\|_{1} \\
\leq 2 \| \mathcal{P}_{\sigma^{a}, \mathcal{N}}^{\dagger} a, b \\
\left.\leq 2 \| a_{2}^{\dagger}\right)\left\|_{\infty}\right\| \boldsymbol{N}_{2}^{\dagger}\left\|_{\infty}\right\| \mathcal{N}^{a, b}\left(\sigma^{a}\right)-\mathcal{N}(\sigma) \|_{1}^{\frac{1}{2}} \operatorname{tr}(\sigma),
\end{aligned}
$$

where the final step uses the Powers-Størmer inequality [32]. The triangle inequality implies that

$$
\begin{aligned}
& \lim _{a \rightarrow \infty} \lim _{b \rightarrow \infty}\left\|\mathcal{N}^{a, b}\left(\sigma^{a}\right)-\mathcal{N}(\sigma)\right\|_{1} \\
& \quad \leq \lim _{a \rightarrow \infty} \lim _{b \rightarrow \infty}\left\|\mathcal{N}^{a, b}\left(\sigma^{a}\right)-\mathcal{N}\left(\sigma^{a}\right)\right\|_{1}+\left\|\mathcal{N}\left(\sigma^{a}\right)-\mathcal{N}(\sigma)\right\|_{1} \\
& \quad \leq \lim _{a \rightarrow \infty}\left\|\sigma^{a}-\sigma\right\|_{1} \\
& \quad=0
\end{aligned}
$$

where the penultimate step uses Grümm's theorem [37, Theorem 2.19].

It remains to argue for the convergence $\lim _{a \rightarrow \infty} \lim _{b \rightarrow \infty} \mathcal{P}_{\sigma^{a}, \mathcal{N}} a, b=\mathcal{P}_{\sigma, \mathcal{N}}$. Consider that (58) implies that

$$
\begin{aligned}
& \lim _{a \rightarrow \infty} \lim _{b \rightarrow \infty}\left\langle\phi^{\prime}\left|\mathcal{N}(\sigma)^{\frac{1}{2}} \mathcal{P}_{\sigma^{a}, \mathcal{N} a, b}^{\dagger}(|\phi\rangle\langle\psi|) \mathcal{N}(\sigma)^{\frac{1}{2}}\right| \psi^{\prime}\right\rangle \\
& \quad=\left\langle\phi^{\prime}\left|\mathcal{N}(\sigma)^{\frac{1}{2}} \mathcal{P}_{\sigma, \mathcal{N}}^{\dagger}(|\phi\rangle\langle\psi|) \mathcal{N}(\sigma)^{\frac{1}{2}}\right| \psi^{\prime}\right\rangle
\end{aligned}
$$


for all $|\phi\rangle,|\psi\rangle \in A$ and $\left|\phi^{\prime}\right\rangle,\left|\psi^{\prime}\right\rangle \in B$. This establishes convergence of $\mathcal{P}_{\sigma^{a}, \mathcal{N}^{a}, b}^{\dagger}$ to $\mathcal{P}_{\sigma, \mathcal{N}}^{\dagger}$ for all $|\phi\rangle,|\psi\rangle \in A$ and $\left|\phi^{\prime}\right\rangle,\left|\psi^{\prime}\right\rangle \in \operatorname{supp}(\mathcal{N}(\sigma))$, which in turn allows us to conclude convergence of $\mathcal{P}_{\sigma^{a}, \mathcal{N}^{a, b}}$ to $\mathcal{P}_{\sigma, \mathcal{N}}$.

Before stating the following lemma, we introduce the shorthand

$$
\mathcal{U}_{\omega, t}: X \mapsto \omega^{\mathrm{i} t} X \omega^{-\mathrm{i} t}
$$

where $\omega$ is a positive semi-definite operator.

Lemma 3.7. For any $\sigma \in \mathrm{P}(A)$, any $\rho \in \mathrm{S}_{\sigma}(A)$, any $\mathcal{N} \in \operatorname{TPCP}(A, B)$, and all $t \in \mathbb{R}$, the following limit holds

$$
\lim _{a \rightarrow \infty} \lim _{b \rightarrow \infty} F\left(\rho^{a},\left(\mathcal{R}_{\sigma^{a}, \mathcal{N} a, b}^{t} \circ \mathcal{N}^{a, b}\right)\left(\rho^{a}\right)\right)=F\left(\rho,\left(\mathcal{R}_{\sigma, \mathcal{N}}^{t} \circ \mathcal{N}\right)(\rho)\right) .
$$

Proof. We start with a reminder of a standard result: Let $\omega$ and $\left\{\omega_{n}\right\}_{n \in \mathbb{N}}$ be positive semi-definite trace-class operators such that $\lim _{n \rightarrow \infty}\left\|\omega_{n}-\omega\right\|_{1}=0$. Then, for any $t \in \mathbb{R}$ and any bounded linear operator $X$, the term $\omega_{n}^{\mathrm{i} t} X \omega_{n}^{-\mathrm{i} t}$ converges to $\omega^{\mathrm{it}} X \omega^{-\mathrm{i} t}$ for $n \rightarrow \infty$ in the weak operator topology. To see this, we note that by Cauchy-Schwarz we have

$$
\begin{aligned}
& \left|\left\langle\varphi,\left(\omega_{n}^{\mathrm{i} t} X \omega_{n}^{-\mathrm{i} t}-\omega^{\mathrm{i} t} X \omega^{-\mathrm{i} t}\right) \phi\right\rangle\right| \\
& \quad=\left|\left\langle\varphi,\left(\omega_{n}^{\mathrm{i} t} X \omega_{n}^{-\mathrm{i} t}-\omega_{n}^{\mathrm{i} t} X \omega^{-\mathrm{i} t}\right)+\left(\omega_{n}^{\mathrm{i} t} X \omega^{-\mathrm{i} t}-\omega^{\mathrm{i} t} X \omega^{-\mathrm{i} t}\right) \phi\right\rangle\right| \\
& \quad \leq\left|\left\langle X \omega_{n}^{-\mathrm{i} t} \varphi,\left(\omega_{n}^{-\mathrm{i} t}-\omega^{-\mathrm{i} t}\right) \phi\right\rangle\right|+\left|\left\langle\left(\omega_{n}^{\mathrm{i} t}-\omega^{\mathrm{i} t}\right) \varphi, X \omega^{-\mathrm{i} t} \phi\right\rangle\right| \\
& \quad \leq\|X\|\|\varphi\|\left\|\left(\omega_{n}^{-\mathrm{i} t}-\omega^{-\mathrm{i} t}\right) \phi\right\|+\|X\|\|\phi\|\left\|\left(\omega_{n}^{\mathrm{i} t}-\omega^{\mathrm{i} t}\right) \varphi\right\| .
\end{aligned}
$$

We first note that for any fixed $k \in \mathbb{N}$

$$
\lim _{n \rightarrow \infty}\left\|\left(\Pi_{k} \omega_{n}^{-i t} \Pi_{k}-\Pi_{k} \omega^{-i t} \Pi_{k}\right) \phi\right\|=0 .
$$

To see this, we recall Duhamel's formula which shows that

$$
\begin{aligned}
& \left(\Pi_{k} \omega_{n}^{-\mathrm{i} t} \Pi_{k}-\Pi_{k} \omega^{-\mathrm{i} t} \Pi_{k}\right) \\
& \quad=\left(\mathrm{e}^{-\mathrm{i} t \log \Pi_{k} \omega_{n} \Pi_{k}}-\mathrm{e}^{-\mathrm{i} t \log \Pi_{k} \omega \Pi_{k}}\right) \\
& \quad=\mathrm{i} \int_{0}^{t} \mathrm{~d} s \mathrm{e}^{-\mathrm{i} s \log \Pi_{k} \omega_{n} \Pi_{k}}\left(\log \Pi_{k} \omega \Pi_{k}-\log \Pi_{k} \omega_{n} \Pi_{k}\right) \mathrm{e}^{-\mathrm{i}(t-s) \log \Pi_{k} \omega \Pi_{k}} .
\end{aligned}
$$

Theorem X.3.7 from [5] ensures that for any fixed $k \in \mathbb{N}$

$$
\begin{aligned}
& \left\|\log \Pi_{k} \omega \Pi_{k}-\log \Pi_{k} \omega_{n} \Pi_{k}\right\| \\
& \quad \leq\left\|\left(\Pi_{k} \omega_{n} \Pi_{k}\right)^{-1}\right\|\left\|\Pi_{k} \omega \Pi_{k}-\Pi_{k} \omega_{n} \Pi_{k}\right\|+O\left(\left\|\Pi_{k} \omega \Pi_{k}-\Pi_{k} \omega_{n} \Pi_{k}\right\|^{2}\right),
\end{aligned}
$$

where $\left\|\left(\Pi_{k} \omega_{n} \Pi_{k}\right)^{-1}\right\|<\infty$ and $\lim _{n \rightarrow \infty}\left\|\Pi_{k} \omega \Pi_{k}-\Pi_{k} \omega_{n} \Pi_{k}\right\|=0$. This justifies (84). By the triangle inequality, we have

$$
\begin{aligned}
& \left\|\left(\omega_{n}^{-i t}-\omega^{-i t}\right) \phi\right\| \\
& \quad=\left\|\left(\omega_{n}^{-i t}-\omega^{-i t}\right)\left(\phi-\Pi_{k} \phi+\Pi_{k} \phi\right)\right\| \\
& \quad \leq\left\|\left(\Pi_{k}-\Pi_{k}+\mathrm{id}\right)\left(\omega_{n}^{-i t} \Pi_{k}-\omega^{-i t} \Pi_{k}\right) \phi\right\|+\left\|\left(\omega_{n}^{-i t}-\omega^{-i t}\right)\left(\phi-\Pi_{k} \phi\right)\right\|
\end{aligned}
$$




$$
\begin{aligned}
\leq & \left.\| \Pi_{k} \omega_{n}^{-\mathrm{i} t} \Pi_{k}-\Pi_{k} \omega^{-\mathrm{i} t} \Pi_{k}\right) \phi\|+\|\left(\mathrm{id}-\Pi_{k}\right)\left(\omega_{n}^{-\mathrm{i} t} \Pi_{k}-\omega^{-\mathrm{i} t} \Pi_{k}\right) \phi \| \\
& +\left\|\left(\omega_{n}^{-\mathrm{i} t}-\omega^{-\mathrm{i} t}\right)\left(\phi-\Pi_{k} \phi\right)\right\|,
\end{aligned}
$$

where for any fixed $n \in \mathbb{N}$

$$
\lim _{k \rightarrow \infty}\left\|\left(\mathrm{id}-\Pi_{k}\right)\left(\omega_{n}^{-\mathrm{i} t} \Pi_{k}-\omega^{-\mathrm{i} t} \Pi_{k}\right) \phi\right\|=0
$$

and

$$
\lim _{k \rightarrow \infty}\left\|\left(\omega_{n}^{-i t}-\omega^{-i t}\right)\left(\phi-\Pi_{k} \phi\right)\right\|=0
$$

We thus conclude that

$$
\begin{aligned}
\lim _{n \rightarrow \infty}\left\|\left(\omega_{n}^{-i t}-\omega^{-i t}\right) \phi\right\| & \leq \lim _{n \rightarrow \infty} \lim _{k \rightarrow \infty}\left\|\left(\Pi_{k} \omega_{n}^{-i t} \Pi_{k}-\Pi_{k} \omega^{-i t} \Pi_{k}\right) \phi\right\| \\
& =\lim _{k \rightarrow \infty} \lim _{n \rightarrow \infty}\left\|\left(\Pi_{k} \omega_{n}^{-i t} \Pi_{k}-\Pi_{k} \omega^{-i t} \Pi_{k}\right) \phi\right\| \\
& =0,
\end{aligned}
$$

where in the penultimate step we swap the order of the limits [33, Theorem 7.11]. The final step uses (84).

We thus have the following convergences in the weak operator topology:

$$
\lim _{a \rightarrow \infty} \lim _{b \rightarrow \infty} \mathcal{U}_{\mathcal{N}^{a, b}\left(\sigma^{a}\right), t}=\mathcal{U}_{\mathcal{N}(\sigma), t} \quad \text { and } \quad \lim _{a \rightarrow \infty} \mathcal{U}_{\sigma^{a}, t}=\mathcal{U}_{\sigma, t} .
$$

The serial concatenation of weakly converging channels converges to the serial concatenation of the limit, so that $\lim _{a \rightarrow \infty} \lim _{b \rightarrow \infty} \mathcal{R}_{\sigma^{a}, \mathcal{N}^{a, b}}^{t} \circ \mathcal{N}^{a, b}=\mathcal{R}_{\sigma, \mathcal{N}}^{t} \circ \mathcal{N}$, where we used the above and Lemma 3.6. Then, we can conclude that the fidelity converges because it is continuous in its inputs (see, e.g., [8, Lemma B.9]), and for our case considered here, convergence in the weak sense implies convergence in the trace norm [14, Chapter 11]. This proves the assertion.

By invoking Theorem 2.1 for finite-dimensional Hilbert spaces ${ }^{6}$ together with Lemmas 3.5 and 3.7, we find for any $\rho \in \mathrm{S}_{\sigma}(A)$

$$
\begin{aligned}
D(\rho \| \sigma)= & \lim _{a \rightarrow \infty} D\left(\rho^{a} \| \sigma^{a}\right) \\
\geq \limsup _{a \rightarrow \infty} \limsup _{b \rightarrow \infty} D\left(\mathcal{N}^{a, b}\left(\rho^{a}\right) \| \mathcal{N}^{a, b}\left(\sigma^{a}\right)\right) & \quad 2 \int_{\mathbb{R}} \mathrm{d} t \beta_{0}(t) \log F\left(\rho^{a},\left(\mathcal{R}_{\sigma^{a}, \mathcal{N}^{a, b}}^{\frac{t}{2}} \circ \mathcal{N}^{a, b}\right)\left(\rho^{a}\right)\right) \\
& \quad D(\mathcal{N}(\rho) \| \mathcal{N}(\sigma))-2 \int_{\mathbb{R}} \mathrm{d} t \beta_{0}(t) \log F\left(\rho,\left(\mathcal{R}_{\sigma, \mathcal{N}}^{\frac{t}{2}} \circ \mathcal{N}\right)(\rho)\right),
\end{aligned}
$$

where we also used Fatou's lemma that ensures

$$
\begin{aligned}
& \liminf _{a \rightarrow \infty} \liminf _{b \rightarrow \infty} \int_{\mathbb{R}} \mathrm{d} t \beta_{0}(t)\left(-2 \log F\left(\rho^{a},\left(\mathcal{R}_{\sigma^{a}, \mathcal{N}^{a, b}}^{\frac{t}{2}} \circ \mathcal{N}^{a, b}\right)\left(\rho^{a}\right)\right)\right) \\
& \geq \int_{\mathbb{R}} \mathrm{d} t \beta_{0}(t) \liminf _{a \rightarrow \infty} \liminf _{b \rightarrow \infty}\left(-2 \log F\left(\rho^{a},\left(\mathcal{R}_{\sigma^{a}, \mathcal{N}^{a, b}}^{\frac{t}{2}} \circ \mathcal{N}^{a, b}\right)\left(\rho^{a}\right)\right)\right) .
\end{aligned}
$$

\footnotetext{
6 Recall that by Remark 3.4, Theorem 2.1 remains valid for subnormalized states and a trace non-increasing completely positive map.
} 
This concludes the proof of Theorem 2.1

\section{Universal and Explicit Refinements of Other Entropy Inequalities}

It is well known (see, e.g., [34]) that the monotonicity of the relative entropy under trace-preserving completely positive maps is closely related to (i) strong subadditivity, (ii) concavity of the conditional entropy, and (iii) joint convexity of the relative entropy. Based on this relation, Remark 2.2 can be used to derive universal and explicit remainder terms for the statements (i)-(iii). We note that universal means that the recovery map that occurs in the remainder term does not depend on all possible parameters. Within this section, we assume that $A$, $B$, and $C$ are finite-dimensional Hilbert spaces. For $n \in \mathbb{N}$, the $n$-simplex is denoted by $\Delta_{n}:=\left\{x \in \mathbb{R}^{n}: x \geq 0, \sum_{i=1}^{n} x_{i}=1\right\}$.

Applying Remark 2.2 for $\sigma=\operatorname{id}_{A} \otimes \rho_{B C} \in \mathrm{P}(A \otimes B \otimes C), \rho=\rho_{A B C} \in$ $\mathrm{S}_{\sigma}(A \otimes B \otimes C)$, and $\mathcal{N}_{A B C \rightarrow A B}=\mathcal{I}_{A B} \otimes \operatorname{tr}_{C}$ implies a strengthened version of the result that has been established in [38, Theorem 2.1, Corollary 2.4, and Remark 2.5].

Corollary 4.1 (Strong subadditivity). For any density operator $\rho_{A B C} \in \mathrm{S}(A \otimes$ $B \otimes C)$, we have

$$
I(A: C \mid B)_{\rho} \geq-2 \log F\left(\rho_{A B C}, \mathcal{R}_{B \rightarrow B C}\left(\rho_{A B}\right)\right),
$$

where $\mathcal{R}_{B \rightarrow B C}(\cdot)=\int_{\mathbb{R}} \mathrm{d} t \beta_{0}(t) \mathcal{R}_{\rho_{B C}, \operatorname{tr}_{C}}(\cdot)$ as defined in Theorem 2.1.

From Corollary 4.1, we can deduce a universal remainder term for the concavity of the conditional entropy. We note that a similar remainder term has been conjectured in [4]. Furthermore, a remainder term that, however, is neither universal nor explicit has been proven in [42, Corollary 12].

Corollary 4.2 (Concavity of conditional entropy). For any $\rho_{A B} \in \mathrm{S}(A \otimes B)$ we have

$$
H(A \mid B)_{\rho}-\sum_{x \in \mathcal{X}} \nu(x) H(A \mid B)_{\rho^{x}} \geq-2 \log \sum_{x \in \mathcal{X}} \nu(x) F\left(\rho_{A B}^{x}, \mathcal{R}_{B \rightarrow A B}\left(\rho_{B}^{x}\right)\right),
$$

for all ensembles $\left\{\nu(x), \rho_{A B}^{x}\right\}_{x \in \mathcal{X}}$ with $\nu \in \Delta_{\mathcal{X}}$ and $\rho_{A B}^{x} \in \mathrm{S}(A \otimes B)$ such that $\rho_{A B}=\sum_{x \in \mathcal{X}} \nu(x) \rho_{A B}^{x}$ where $\mathcal{R}_{B \rightarrow A B}(\cdot)=\int_{\mathbb{R}} \mathrm{d} t \beta_{0}(t) \mathcal{R}_{\rho_{A B}, \operatorname{tr}_{A}}(\cdot)$ as defined in Theorem 2.1.

Proof. Let us consider the following classical-quantum state

$$
\phi_{X A B}=\sum_{x \in \mathcal{X}} \nu(x)|x\rangle\left\langle\left. x\right|_{X} \otimes \rho_{A B}^{x} .\right.
$$

By Corollary 4.1 and a simple fact ensuring that the fidelity for orthogonal input states decomposes (see Lemma A.1 in [38]), we find 


$$
\begin{aligned}
H(A \mid B)_{\rho}-\sum_{x \in \mathcal{X}} \nu(x) H(A \mid B)_{\rho^{x}} & =H(A \mid B)_{\phi}-H(A \mid B X)_{\phi} \\
& =I(X: A \mid B)_{\phi} \\
& \geq-2 \log F\left(\phi_{X A B}, \mathcal{R}_{B \rightarrow A B}\left(\phi_{X B}\right)\right) \\
& =-2 \log \sum_{x \in \mathcal{X}} \nu(x) F\left(\rho_{A B}^{x}, \mathcal{R}_{B \rightarrow A B}\left(\rho_{B}^{x}\right)\right) .
\end{aligned}
$$

We note that by Corollary 4.1 the recovery map $\mathcal{R}_{B \rightarrow A B}$ is explicit and universal in the sense that it only depends on $\rho_{A B}$.

Remark 2.2 implies a universal remainder term for the joint convexity of the relative entropy. We note that a similar remainder term has been conjectured in [35]. Corollary 13 in [42] proves such a remainder term that, however, is neither universal nor explicit.

Corollary 4.3 (Joint convexity of relative entropy). For any $\nu \in \Delta_{\mathcal{X}}$, $\left\{\sigma^{x}\right\}_{x \in \mathcal{X}}$ with $\sigma^{x} \in \mathrm{P}(A)$ for all $x \in \mathcal{X}, \sigma_{X A}=\sum_{x \in \mathcal{X}} \nu(x)|x\rangle\left\langle\left. x\right|_{X} \otimes \sigma^{x}\right.$, any $\rho_{x} \in \mathrm{S}_{\sigma^{x}}(A)$ and $\rho=\sum_{x \in \mathcal{X}} \nu(x) \rho^{x}$ we have

$$
\sum_{x \in \mathcal{X}} \nu(x) D\left(\rho^{x} \| \sigma^{x}\right)-D(\rho \| \sigma) \geq-2 \log \sum_{x \in \mathrm{S}} \nu(x) F\left(\rho^{x}, \mathcal{R}_{\sigma_{X A}, \operatorname{tr}_{X}}(\rho)\right),
$$

where $\mathcal{R}_{\sigma_{X A}, \operatorname{tr}_{X}}(\cdot)=\int_{\mathbb{R}} \mathrm{d} t \beta_{0}(t) \mathcal{R}_{\sigma_{X A}, \operatorname{tr}_{X}}^{t}(\cdot)$ as defined in Theorem 2.1 .

Proof. Let us consider the states

$$
\rho_{X A}:=\sum_{x \in \mathcal{X}} \nu(x)|x\rangle\left\langle\left. x\right|_{X} \otimes \rho^{x} \quad \text { and } \quad \rho=\rho_{A}=\sum_{x \in \mathcal{X}} \nu(x) \rho^{x} .\right.
$$

Since the relative entropy decomposes for orthogonal input states (see Lemma B.2 in [38]), Remark 2.2 implies that the recovery map $\mathcal{R}_{\sigma_{X A}, \operatorname{tr}_{X}}(\cdot)=$ $\int_{\mathbb{R}} \mathrm{d} t \beta_{0}(t) \mathcal{R}_{\sigma_{X A}, \operatorname{tr}_{X}}^{t}(\cdot)$ satisfies

$$
\begin{aligned}
\sum_{x \in \mathcal{X}} \nu(x) D\left(\rho^{x} \| \sigma^{x}\right)-D(\rho \| \sigma) & =D\left(\rho_{X A} \| \sigma_{X A}\right)-D\left(\rho_{A} \| \sigma_{A}\right) \\
& \geq-2 \log F\left(\rho_{X A}, \mathcal{R}_{\sigma_{X A}, \operatorname{tr}_{X}}(\rho)\right) \\
& =-2 \log \sum_{x \in \mathcal{X}} \nu(x) F\left(\rho^{x}, \mathcal{R}_{\sigma_{X A}, \operatorname{tr}_{X}}(\rho)\right),
\end{aligned}
$$

where the final step uses that the fidelity for orthogonal input states decomposes (see Lemma A.1 in [38]).

\section{Approximate Quantum Error Correction}

Theorem 2.1 allows for establishing an alternate, information-theoretic set of conditions for approximate quantum error correction. Recall that in quantum error correction the main goal is to protect a set of states from the action of a noisy quantum channel, by encoding this set into a subspace of a given Hilbert space. The quantum error correction conditions given in the seminal 
works $[3,18]$ are necessary and sufficient for perfect quantum error correction. Although these works were essential for gaining an understanding of quantum error correction, the conditions given represent a strong idealization, since we can never hope in practice to have perfect quantum error correction. So this realization motivated some follow-up works on approximate quantum error correction (see $[2,25]$ and references therein), in which one seeks to determine conditions under which approximate recovery from the action of a given noisy channel is possible. We mention that some of these works made essential use of the Petz recovery map.

In [12], information-theoretic conditions for perfect error correction were given in terms of the quantum relative entropy. We recall their result: Let $A$ be a Hilbert space, let $C$ be a subspace of $A$ (called the "codespace"), and let $\Pi$ denote the projection onto $C$. Then

$$
\{\forall \rho \in \mathrm{S}(C): D(\rho \| \Pi)=D(\mathcal{N}(\rho) \| \mathcal{N}(\Pi))\} \Longleftrightarrow\left\{\forall \rho \in \mathrm{S}(C): \rho=\left(\mathcal{P}_{\Pi, \mathcal{N}} \circ \mathcal{N}\right)(\rho)\right\},
$$

where $\mathcal{P}_{\Pi, \mathcal{N}}(\cdot)$ is the Petz recovery map defined in (5). So perfect error correction is possible if and only if for all $\rho \in \mathrm{S}(C)$ the pairwise distinguishability of $\rho$ with $\Pi$ does not decrease under the action of the noisy channel $\mathcal{N}$.

Given the statement in (113) and the prior work on approximate quantum error correction, it is natural to wonder whether a robust version of (113) exists. Indeed, Theorem 2.1 (or rather Remark 2.2) implies such a statement, establishing necessary and sufficient information-theoretic conditions for approximate quantum error correction. Loosely speaking, we can now say that approximate error correction is possible if and only if the pairwise distinguishability of $\rho$ with $\Pi$ does not decrease by too much under the action of the noisy channel $\mathcal{N}$ for all $\rho \in \mathrm{S}(C)$. The corollary below is a consequence of Remark 2.2 and some other known facts.

Corollary 5.1. Let $\varepsilon \in[0,1], A$ and $B$ be finite-dimensional Hilbert spaces, $C$ be a subspace of $A$ (called the "codespace"), $\mathcal{N} \in \operatorname{TPCP}(A, B)$ a quantum channel, and let $\Pi$ denote the projection onto $C$. If for all $\rho \in \mathrm{S}(C)$ we have

$$
D(\rho \| \Pi)-D(\mathcal{N}(\rho) \| \mathcal{N}(\Pi)) \leq \varepsilon,
$$

then it is possible to approximately recover every state $\rho \in \mathrm{S}(C)$, in the sense that we have for all $\rho \in \mathrm{S}(C)$

$$
F\left(\rho,\left(\mathcal{R}_{\Pi, \mathcal{N}} \circ \mathcal{N}\right)(\rho)\right) \geq 1-\frac{1}{2} \varepsilon .
$$

Conversely, if (115) holds for $\varepsilon \in[0,1]$, then we have for all $\rho \in \mathrm{S}(C)$

$$
D(\rho \| \Pi)-D(\mathcal{N}(\rho) \| \mathcal{N}(\Pi)) \leq \sqrt{\varepsilon} \log (\operatorname{dim} C)+h_{2}(\sqrt{\varepsilon}),
$$

where $h_{2}(p):=-p \log p-(1-p) \log (1-p)$ is the binary entropy, with the property that $\lim _{p \searrow 0} h_{2}(p)=0$.

Proof. The first statement is a direct consequence of Remark 2.2, found by setting $\rho=\rho, \sigma=\Pi$, and $\mathcal{N}=\mathcal{N}$ and then applying the inequality $-\log (x) \geq$ $1-x$, which holds for $x \in[0,1]$. 
To prove the second statement, suppose that (115) holds. By the Fuchsvan de Graaf inequality [9], (115) implies that for all $\rho \in \mathrm{S}(C)$ we have

$$
\frac{1}{2}\left\|\rho-\left(\mathcal{R}_{\Pi, \mathcal{N}} \circ \mathcal{N}\right)(\rho)\right\|_{1} \leq \sqrt{\varepsilon} .
$$

Then, consider the following chain of inequalities, which holds for all $\rho \in \mathrm{S}(C)$ :

$$
\begin{aligned}
D & (\rho \| \Pi)-D(\mathcal{N}(\rho) \| \mathcal{N}(\Pi)) \\
& \leq D(\rho \| \Pi)-D\left(\left(\mathcal{R}_{\Pi, \mathcal{N}} \circ \mathcal{N}\right)(\rho) \|\left(\mathcal{R}_{\Pi, \mathcal{N}} \circ \mathcal{N}\right)(\Pi)\right) \\
& =D(\rho \| \Pi)-D\left(\left(\mathcal{R}_{\Pi, \mathcal{N}} \circ \mathcal{N}\right)(\rho) \| \Pi\right) \\
& =-H(\rho)+H\left(\left(\mathcal{R}_{\Pi, \mathcal{N}} \circ \mathcal{N}\right)(\rho)\right)+\operatorname{tr}\left(\left[\left(\mathcal{R}_{\Pi, \mathcal{N}} \circ \mathcal{N}\right)(\rho)-\rho\right] \log \Pi\right) \\
& \leq \sqrt{\varepsilon} \log \operatorname{dim}(C)+h_{2}(\sqrt{\varepsilon}) .
\end{aligned}
$$

The first inequality is a consequence of monotonicity of quantum relative entropy with respect to the recovery map $\mathcal{R}_{\Pi, \mathcal{N}}$. The first equality follows because $\left.\left(\mathcal{R}_{\Pi, \mathcal{N}} \circ \mathcal{N}\right)(\Pi)\right)=\Pi$. The second equality is a rewriting, and the last inequality follows from the Fannes-Audenaert inequality [1] (see also [43, Lemma 1]) and the facts that $\operatorname{supp}(\rho) \subseteq \operatorname{supp}(\Pi), \operatorname{supp}\left(\left(\mathcal{R}_{\Pi, \mathcal{N}} \circ \mathcal{N}\right)(\rho)\right) \subseteq$ $\operatorname{supp}(\Pi)$, and $\log \Pi=0$ on the support of $\Pi$.

\section{Conclusion}

In this work, we showed that for any nonnegative operator $\sigma$ and for any channel $\mathcal{N}$ there exists an explicit and universal recovery map $\mathcal{R}_{\sigma, \mathcal{N}}$ such that

$$
D(\rho \| \sigma)-D(\mathcal{N}(\rho) \| \mathcal{N}(\sigma)) \geq-2 \log F\left(\rho,\left(\mathcal{R}_{\sigma, \mathcal{N}} \circ \mathcal{N}\right)(\rho)\right)
$$

for all density operators $\rho$ such that $\operatorname{supp}(\rho) \subseteq \operatorname{supp}(\sigma)$. A consequence of its universality is that the recovery map $\mathcal{R}_{\sigma, \mathcal{N}}$ satisfies $\left(\mathcal{R}_{\sigma, \mathcal{N}} \circ \mathcal{N}\right)(\sigma)=\sigma$. The present work thus constitutes an improvement over [42] since the recovery map is constructed explicitly and is independent of $\rho$. Knowing the explicit structure of the recovery map that satisfies (122) can be helpful in various scenarios. For example, if this is to be implemented by experimentalists or in an actual quantum computer it is helpful to have an explicit recovery map. We showed that the inequality (122) implies universal and explicit remainder terms for other entropy inequalities such as strong subadditivity, concavity of the entropy, and the joint convexity of the relative entropy. Furthermore, it can be useful in the context of quantum sufficient statistics and approximate quantum error correction.

\section{Acknowledgements}

We thank Omar Fawzi, Rupert Frank, Jürg Fröhlich, Elliott Lieb, Volkher Scholz, and Marco Tomamichel for helpful discussions. We further thank the anonymous referee for constructive feedback. MJ's work was supported by NSF DMS 1501103 and NSF-DMS 1201886. RR and DS acknowledge support by the European Research Council (ERC) via Grant No. 258932, by 
the Swiss National Science Foundation (SNSF) via the National Centre of Competence in Research "QSIT," and by the European Commission via the project "RAQUEL." MMW acknowledges support from the NSF under Award Nos. CCF-1350397 and 1714215 and the DARPA Quiness Program through US Army Research Office award W31P4Q-12-1-0019. He is also grateful to co-authors MJ, RR, and AW for hospitality and support during research visits in summer 2015. AWs work was supported by the EU (STREP RAQUEL), the ERC (AdG IRQUAT), the Spanish MINECO (Grant FIS2013-40627-P) with the support of FEDER funds, as well as by the Generalitat de Catalunya CIRIT, Project 2014-SGR-966.

Open Access. This article is distributed under the terms of the Creative Commons Attribution 4.0 International License (http://creativecommons.org/licenses/ by/4.0/), which permits unrestricted use, distribution, and reproduction in any medium, provided you give appropriate credit to the original author(s) and the source, provide a link to the Creative Commons license, and indicate if changes were made.

\section{Appendix}

\section{Appendix A. Proof of Lemma 3.2}

We begin by recalling Hirschman's strengthening [13] of the Hadamard threeline theorem (see, e.g., [10, Lemma 1.3.8]):

Lemma A.1. Let $S:=\{z \in \mathbb{C}: 0 \leq \operatorname{Re}\{z\} \leq 1\}$ and let $g(z)$ be holomorphic on the interior of $S$ and continuous on the boundary, such that

$$
\sup _{z \in S} \exp \{-a|\operatorname{Im} z|\} \log |g(z)| \leq A<\infty,
$$

for some fixed $A$ and $a<\pi$. Then, for $\theta \in(0,1)$, the following bound holds

$$
\log |g(\theta)| \leq \int_{\mathbb{R}} \mathrm{d} t\left(\alpha_{\theta}(t) \log \left(|g(\mathrm{i} t)|^{1-\theta}\right)+\beta_{\theta}(t) \log \left(|g(1+\mathrm{i} t)|^{\theta}\right)\right),
$$

where $\alpha_{\theta}(t)$ and $\beta_{\theta}(t)$ are defined in $(28)$.

We can now prove Lemma 3.2.

Proof of Lemma 3.2. The proof of this theorem is well known, but we provide it for completeness. For fixed $\theta \in(0,1)$, let $q_{\theta}$ be the Hölder conjugate of $p_{\theta}$, defined by

$$
\frac{1}{p_{\theta}}+\frac{1}{q_{\theta}}=1
$$

Similarly, let $q_{0}$ and $q_{1}$ be Hölder conjugates of $p_{0}$ and $p_{1}$, respectively. We can find an operator $X$ such that

$$
\|X\|_{q_{\theta}}=1 \quad \text { and } \quad \operatorname{tr}(X G(\theta))=\|G(\theta)\|_{p_{\theta}} .
$$


We can write the singular value decomposition for $X$ in the form $X=U D^{1 / q_{\theta}} V$ (implying $\operatorname{tr}(D)=1$ ). For $z \in S$, define

$$
X(z):=U D^{\frac{1-z}{q_{0}}+\frac{z}{q_{1}}} V .
$$

As a consequence, $X(z)$ is holomorphic on the interior of $S$ and continuous on the boundary. Also, observe that $X(\theta)=X$. Then, the following function satisfies the requirements needed to apply Lemma A.1:

$$
g(z):=\operatorname{tr}(X(z) G(z))
$$

Indeed, we have that

$$
\log \|G(\theta)\|_{p_{\theta}}=\log |g(\theta)| \leq \int_{\mathbb{R}} \mathrm{d} t\left(\alpha_{\theta}(t) \log \left(|g(\mathrm{i} t)|^{1-\theta}\right)+\beta_{\theta}(t) \log \left(|g(1+\mathrm{i} t)|^{\theta}\right)\right) .
$$

Now, from applying Hölder's inequality and the facts that $\|X(i t)\|_{q_{0}}=1=$ $\|X(1+\mathrm{i} t)\|_{q_{1}}$, we find that

$$
|g(\mathrm{i} t)|=|\operatorname{tr}(X(\mathrm{i} t) G(\mathrm{i} t))| \leq\|X(\mathrm{i} t)\|_{q_{0}}\|G(\mathrm{i} t)\|_{p_{0}}=\|G(\mathrm{i} t)\|_{p_{0}}
$$

and

$$
|g(1+\mathrm{i} t)|=|\operatorname{tr}(X(1+\mathrm{i} t) G(1+\mathrm{i} t))| \leq\|X(1+\mathrm{i} t)\|_{q_{1}}\|G(1+\mathrm{i} t)\|_{p_{1}}=\|G(1+\mathrm{i} t)\|_{p_{1}} .
$$

Bounding (129) from above using these inequalities then gives (27).

\section{References}

[1] Audenaert, K.M.R.: A sharp continuity estimate for the von Neumann entropy. J. Phys. A Math. Theor. 40(28), 8127 (2007)

[2] Barnum, H., Knill, E.: Reversing quantum dynamics with near-optimal quantum and classical fidelity. J. Math. Phys. 43(5), 2097-2106 (2002). arXiv:quant-ph/0004088

[3] Bennett, C.H., DiVincenzo, D.P., Smolin, J.A., Wootters, W.K.: Mixed-state entanglement and quantum error correction. Phys. Rev. A 54(5), 3824-3851 (1996)

[4] Berta, M., Lemm, M., Wilde, M.M.: Monotonicity of quantum relative entropy and recoverability. Quantum Inf. Comput. 15(15 \& 16), 1333-1354 (2015)

[5] Bhatia, R.: Matrix Analysis. Springer, Berlin (1997)

[6] Brandão, F.G.S.L., Harrow, A.W., Oppenheim, J., Strelchuk, S.: Quantum conditional mutual information, reconstructed states, and state redistribution. Phys. Rev. Lett. 115(5), 050501 (2015)

[7] Devetak, I., Yard, J.: Exact cost of redistributing multipartite quantum states. Phys. Rev. Lett. 100(23), 230501 (2008)

[8] Fawzi, O., Renner, R.: Quantum conditional mutual information and approximate Markov chains. Commun. Math. Phys. 340(2), 575-611 (2015)

[9] Fuchs, C., van de Graaf, J.: Cryptographic distinguishability measures for quantum-mechanical states. IEEE Trans. Inf. Theory 45(4), 1216-1227 (1999)

[10] Grafakos, L.: Classical Fourier Analysis, 2nd edn. Springer, Berlin (2008)

[11] Grümm, H.: Two theorems about $C_{p}$. Rep. Math. Phys. 4(3), 211-215 (1973) 
[12] Hiai, F., Mosonyi, M., Petz, D., Beny, C.: Quantum f-divergences and error correction. Rev. Math. Phys. 23(7), 691-747 (2011). arXiv:1008.2529

[13] Hirschman, I.I.: A convexity theorem for certain groups of transformations. Journal d'Analyse Mathématique 2(2), 209-218 (1952)

[14] Holevo, A.S.: Quantum Systems, Channels, Information. De Gruyter Studies in Mathematical Physics 16, de Gruyter (2012)

[15] Jencǒvà, A.: Randomization Theorems for Quantum Channels. arXiv:1404.3900 (2014)

[16] Jencǒvà, A., Petz, D.: Sufficiency in quantum statistical inference. Commun. Math. Phys. 263(1), 259-276 (2006)

[17] Jencǒvà, A., Petz, D.: Sufficiency in quantum statistical inference: a survey with examples. Infin. Dimens. Anal. Quantum Probab. Relat. Top. 09(03), 331-351 (2006)

[18] Knill, E., Laflamme, R.: Theory of quantum error-correcting codes. Phys. Rev. A 55(2), 900-911 (1997)

[19] Li, K., Winter, A.: Squashed Entanglement, k-Extendibility, Quantum Markov Chains, and Recovery Maps. Found. Phys. (2018). https://doi.org/10.1007/ s10701-018-0143-6

[20] Lieb, E.H., Ruskai, M.B.: A fundamental property of quantum-mechanical entropy. Phys. Rev. Lett. 30, 434-436 (1973)

[21] Lieb, E.H., Ruskai, M.B.: Proof of the strong subadditivity of quantummechanical entropy. J. Math. Phys. 14(12), 1938-1941 (1973)

[22] Lindblad, G.: Entropy, information and quantum measurements. Commun. Math. Phys. 33, 305-322 (1973)

[23] Lindblad, G.: Expectations and entropy inequalities for finite quantum systems. Commun. Math. Phys. 39(2), 111-119 (1974)

[24] Lindblad, G.: Completely positive maps and entropy inequalities. Commun. Math. Phys. 40(2), 147-151 (1975)

[25] Mandayam, P., Ng, H.K.: Towards a unified framework for approximate quantum error correction. Phys. Rev. A 86(1), 012335 (2012). arXiv:1202.5139

[26] Mosonyi, M., Petz, D.: Structure of sufficient quantum coarse-grainings. Lett. Math. Phys. 68(1), 19-30 (2004)

[27] Nielsen, M.A., Chuang, I.L.: Quantum Computation and Quantum Information. Cambridge University Press, Cambridge (2000)

[28] Ohya, M., Petz, D.: Quantum Entropy and Its Use. Springer, Berlin (1993)

[29] Petz, D.: Sufficient subalgebras and the relative entropy of states of a von Neumann algebra. Commun. Math. Phys. 105(1), 123-131 (1986)

[30] Petz, D.: Sufficiency of channels over von Neumann algebras. Q. J. Math. 39(1), 97-108 (1988)

[31] Petz, D.: Monotonicity of quantum relative entropy revisited. Rev. Math. Phys. 15(01), 79-91 (2003)

[32] Powers, R.T., Størmer, E.: Free states of the canonical anticommutation relations. Commun. Math. Phys. 16(1), 1-33 (1970)

[33] Rudin, W.: Principles of Mathematical Analysis. International Series in Pure and Applied Mathematics, 3rd edn. McGraw-Hill Book Co, New York (1976) 
[34] Ruskai, M.B.: Inequalities for quantum entropy: a review with conditions for equality. J. Math. Phys. 43(9), 4358-4375 (2002)

[35] Seshadreesan, K.P., Berta, M., Wilde, M.M.: Rényi squashed entanglement, discord, and relative entropy differences. J. Phys. A Math. Theor. 48(39), 395303 (2015)

[36] Shirokov, M.E.: Measures of quantum correlations in infinite-dimensional systems. Sbornik Math. 207(5), 724 (2015). arXiv:1506.06377

[37] Simon, B.: Trace Ideals and Their Applications, 2nd edn. American Mathematical Society, Providence (2005)

[38] Sutter, D., Fawzi, O., Renner, R.: Universal recovery map for approximate Markov chains. Proc. R. Soc. Lond. A Math. Phys. Eng. Sci. 472, 2186 (2016)

[39] Sutter, D., Tomamichel, M., Harrow, A.W.: Strengthened monotonicity of relative entropy via pinched Petz recovery map. IEEE Trans. Inf. Theory 62(5), 2907-2913 (2016)

[40] Uhlmann, A.: The "transition probability" in the state space of a *-algebra. Rep. Math. Phys. 9(2), 273-279 (1976)

[41] Uhlmann, A.: Relative entropy and the Wigner-Yanase-Dyson-Lieb concavity in an interpolation theory. Commun. Math. Phys. 54(1), 21-32 (1977)

[42] Wilde, M.M.: Recoverability in quantum information theory. Proc. R. Soc. Lond. A Math. Phys. Eng. Sci. 471(2182), 20150338 (2015)

[43] Winter, A.: Tight uniform continuity bounds for quantum entropies: conditional entropy, relative entropy distance and energy constraints. Commun. Math. Phys. 347(1), 291-313 (2016)

[44] Winter, A., Li, K.: A Stronger Subadditivity Relation? With Applications to Squashed Entanglement, Sharability and Separability. http://www.scribd.com/ document/337859204 (2012)

Marius Junge

Department of Mathematics

University of Illinois at Urbana-Champaign

Urbana, IL 61801-2975

USA

e-mail: mariusjunge@gmail.com

Renato Renner and David Sutter

Institute for Theoretical Physics

ETH Zurich

Zurich

Switzerland

e-mail: renner@ethz.ch; sutterd@itp.phys.ethz.ch 
Mark M. Wilde

Department of Physics and Astronomy, Center for Computation and Technology, Hearne Institute for Theoretical Physics

Louisiana State University

Baton Rouge, LA 70803

USA

e-mail: mwilde@lsu.edu

Andreas Winter

ICREA \& Física Teòrica: Informació i Fenòmens Quàntics

Universitat Autònoma de Barcelona

08193 Bellaterra, Barcelona

Spain

e-mail: andreas.winter@uab.cat

Communicated by David Perez-Garcia.

Received: August 11, 2017.

Accepted: June 13, 2018. 